Bangladesh J. Plant Taxon. 25(2): 187-207, 2018 (December)

(C) 2018 Bangladesh Association of Plant Taxonomists

\title{
AN ANNOTATED CHECKLIST OF THE ANGIOSPERMIC FLORA OF RAJKANDI RESERVE FOREST OF MOULVIBAZAR, BANGLADESH
}

\author{
A.K.M. Kamrul Haque ${ }^{1}$, Saleh Ahammad Khan, Sarder Nasir Uddin ${ }^{2}$ \\ and Shayla Sharmin SHEtu \\ Department of Botany, Jahangirnagar University, Savar, Dhaka 1342, Bangladesh
}

Keywords: Checklist; Angiosperms; Rajkandi Reserve Forest; Moulvibazar.

\begin{abstract}
This study was carried out to provide the baseline data on the composition and distribution of the angiosperms and to assess their current status in Rajkandi Reserve Forest of Moulvibazar, Bangladesh. The study reports a total of 549 angiosperm species belonging to 123 families, $98(79.67 \%)$ of which consisting of 418 species under 316 genera belong to Magnoliopsida (dicotyledons), and the remaining $25(20.33 \%)$ comprising 132 species of 96 genera to Liliopsida (monocotyledons). Rubiaceae with 30 species is recognized as the largest family in Magnoliopsida followed by Euphorbiaceae with 24 and Fabaceae with 22 species; whereas, in Lilliopsida Poaceae with 32 species is found to be the largest family followed by Cyperaceae and Araceae with 17 and 15 species, respectively. Ficus is found to be the largest genus with 12 species followed by Ipomoea, Cyperus and Dioscorea with five species each. Rajkandi Reserve Forest is dominated by the herbs (284 species) followed by trees (130 species), shrubs (125 species), and lianas (10 species). Woodlands are found to be the most common habitat of angiosperms. A total of 387 species growing in this area are found to be economically useful. 25 species listed in Red Data Book of Bangladesh under different threatened categories are found under Lower Risk (LR) category in this study area.
\end{abstract}

\section{Introduction}

Rajkandi Reserve Forest (RRF) is located in Kamalganj upazilla under Moulvibazar district of Bangladesh. This forest area consists of ca. 2,450 hectares' land of Rajkandi forest range that lies between the $24^{\circ} 12^{\prime}-24^{\circ} 17^{\prime} \mathrm{N}$ and $91^{\circ} 51^{\prime}-91^{\circ} 55^{\prime} \mathrm{E}$, and comprises diverse habitats and ecosystems. This tropical semi-evergreen forest falls within the Indo-Burma hot-spot of biodiversity (Myers et al., 2000).

The extensive floristic exploration throughout British India conducted by J.D. Hooker (18721897) included the Sylhet region of the present political boundary of Bangladesh. Later, David Prain (1903) covered different regions of Bangladesh including Sylhet under his floristic exploration. Kanjilal et al. (1934, 1938-1940) included some areas of Sylhet region too. In those studies, any specific or detail information on local distribution and voucher specimens of the taxa described are missing. Later on, various sporadic inventories have been completed in different areas of greater Sylhet region, such as Das (1968), Alam (1988), Arefin et al. (2011), Uddin and Hassan (2004) and Sobuj and Rahman (2011). However, the flora and plant diversity of Moulvibazar district, have not yet been explored, except the plant diversity (Uddin and Hassan, 2010) or a plant group (Haque et al., 2016) of a particular area. Taxonomic data on the current floristic composition of RRF collected through field inventories and examination of representative plant specimens are still lacking, though such data are important for the sustainable use and

\footnotetext{
${ }^{1}$ Corresponding author. Email: kamrulhaque1234@gmail.com

${ }^{2}$ Bangladesh National Herbarium, Zoo Road, Mirpur-1, Dhaka 1216, Bangladesh.
} 
conservation of plant resources and resource-based development of the area. Therefore, an inventory on the floristic composition of RRF was conducted with the objectives to produce an annotated checklist of the angiospermic species of the area; to determine the current status of threatened species of angiosperms from Bangladesh in this forest; and to collect and preserve representative plant specimens for future reference.

\section{Materials and Methods}

Taxonomic inventories were conducted during 2010 to 2015 through 25 field trips in different seasons throughout the study area (Fig. 1). Necessary field data and representative plant specimens were collected and preserved following standard herbarium techniques (Bridson and Forman, 1989; Singh and Subramaniam, 2008). All plant specimens were preliminarily identified through consulting the experts and matching with relevant voucher specimens preserved at Jahangirnagar University Herbarium (JUH), and Bangladesh National Herbarium (DACB). Some critical specimens were identified at Central National Herbarium, Howrah, India (CAL) during the visit of one of the authors.

The identification of the plant specimens were verfied by matching with the images of pertinent type specimens available in the websites of international herbaria and consulting taxonomic descriptions and keys available in the relevant literatures (Hooker, 1872-1897; Prain, 1903; Wu and Raven, 1994-2001; Wu et al., 1999-2013).

Nomenclature of each taxon was verified following Flora of China (Wu and Raven, 19942001; Wu et al., 1999-2013) and the nomenclatural databases of The Plant List (2013) and TROPICOS (2017). The common names have been cited based on Huq (1986), Pasha and Uddin (2013) and interview with the local people. The families have been arranged following Cronquist (1981), and the genera and species under each family have been arranged alphabetically (Table 1). The economic uses of the species were recorded through interviews with the local people during the field surveys, and consulting the relevant literatures (e.g., Ghani, 1998; van Valkenburg and Bunyapraphatsara, 2002). Status of threatened plant species listed in Red Data Book of Bangladesh (Khan et al., 2001; Ara et al., 2013) was asseesed in context to RRF through field observation on natural distribution and regeneration of each species throughout the area, and IUCN threatened category was estimated consulting IUCN (2001). The voucher specimens have been preserved at JUH and DACB.

\section{Results and Discussion}

A total of 549 species of angiosperms under 412 genera and 123 families have been recorded from RRF with their natural distribution (Table 1). Among these families, 98 (79.67\%) representing 316 genera and 418 species are identified as dicotyledons (Magnoliopsida), whereas, only $25(20.33 \%)$ families consisting of 96 genera and 132 species as monocotyledons (Liliopsida). Among these families, 46 are represented by single species each and only 10 families by more than 10 (10-33) species. This reserve forest is dominated by the herbs comprising 284 species that are followed by trees of 130 species, shrubs of 125 species, and lianas of 10 species. These data indicate that Rajkandi Reserve Forest is floristically rich.

In Magnoliopsida, Rubiaceae with 30 species of 21 genera is recognized as the largest family in RRF followed by Euphorbiaceae with 24 species of 19 genera and Fabaceae with 22 species belonging to 17 genera. Poaceae consisting of 32 species of 25 genera is found to be the largest family in Lilliopsida, followed by Cyperaceae with 17 species of nine genera and Araceae with 15 species of 13 genera. Ficus with 12 species is found as the largest genus in the area, which is 
followed by Ipomoea, Cyperus and Dioscorea with five species each and Maesa, Piper, Senna, Terminalia, Phyllanthus, Mussaenda, Ixora and Bambusa with four species each.
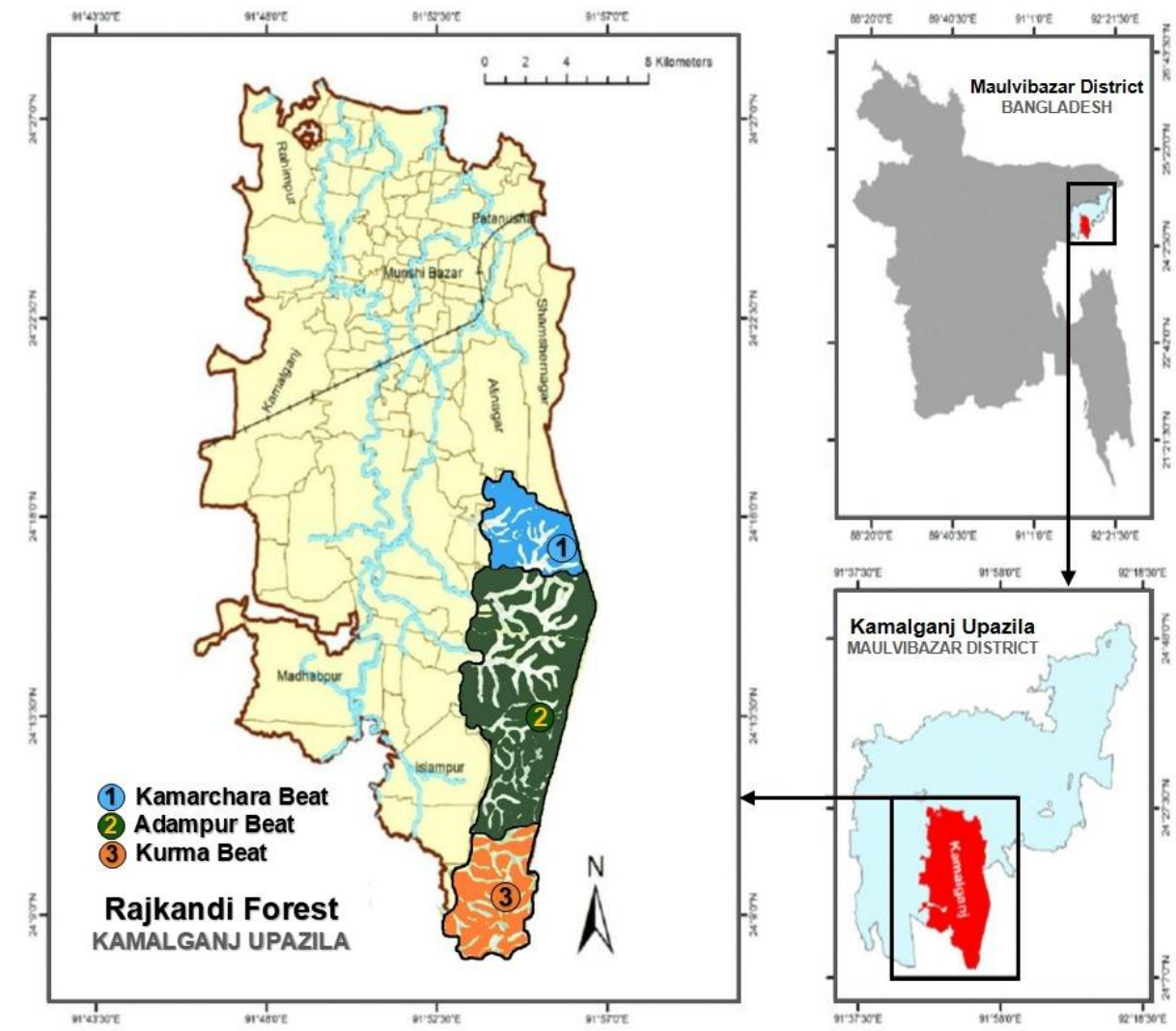

Fig. 1. Rajkandi Forest Range, Kamalganj, Moulvibazar, Bangladesh. (Source: Modified from Haque et al., 2016).

The composition and distribution of species in all of the three forest beats of RRF, namely Adampur, Kurma and Kamarchara, were found to be variable remarkebly. A total of 231, 26 and five species were found to occur exclusively in Adampur-, Kamarchara- and Kurma beats, respectively. The occurance of total 538 species in Adampur beat, with 231 species exclusive and additional 307 species overlapping in other two beats (240 in Kurma and Kamarchara, 46 in Kurma and 21 in Kamarchara), indicates that this forest beat could be considered as a hotspot of biodiversity.

In this forest, woodlands were found to be the most common habitats harbouring the highest number of species (157 species), and this might be due to accumulation of nutrient components and humus-rich soil. In contrast, the finding of hill top to harbour relatively lower number of species (16 species) might be due to their poor humus and nutrient components.

The total number of angiosperm species (549) found in Rajkanndi Reserve Forest during this study is $15.20 \%$ of the total 3,611 species, and that of angiosperm families (123) is $59.42 \%$ of the total 207 families reported for Bangladesh (Ahmed et al., 2008-2009). 
Table 1. List of angiosperm species of Ranjkandi Reserve Forest under Maulvibazar district of Bangladesh.

\begin{tabular}{|c|c|c|c|c|c|c|}
\hline$\underline{\text { Scientific name }}$ & Bangla name & Habit & Habitat & Distrib. & Uses & RSE \\
\hline \multicolumn{7}{|l|}{ MAGNOLIOPSIDA Brongn. } \\
\hline \multicolumn{7}{|l|}{ MAGNOLIACEAE Juss. } \\
\hline Magnolia champaca (L.) Baill. ex Pierre & Champa & Tree, $\mathrm{m}$ & wd & All beats & $\mathrm{T}$ & Kamrul 594 (JUH) \\
\hline \multicolumn{7}{|c|}{ ANNONACEAE Juss. } \\
\hline Annona squamosa $\mathrm{L}$. & Ata & Tree, $\mathrm{s}$ & $\mathrm{ml}(\mathrm{cu})$ & Ad & Fr & Kamrul $2140(\mathrm{JUH})$ \\
\hline A. reticulata $\mathrm{L}$. & Nona & Tree, $\mathrm{s}$ & $\mathrm{ml}(\mathrm{cu})$ & All beats & $\mathrm{Fr}$ & Kamrul 2085 (JUH) \\
\hline $\begin{array}{l}\text { Alphonsea lutea (Roxb.) Hook.f. \& } \\
\text { Thomson }\end{array}$ & Fonseti & Tree, $\mathrm{s}$ & wd & Ad & $\mathrm{Fr}$ & $\begin{array}{l}\text { S.N.Uddin N4625 } \\
\text { (DACB) }\end{array}$ \\
\hline $\begin{array}{l}\text { Dasymaschalon longiflorum (Roxb.) } \\
\text { Finet \& Gagnep. * }\end{array}$ & Kulla & Shrub & wd & All beats & - & Kamrul 707 (JUH) \\
\hline Fissistigma bicolor (Roxb.) Merr. & Hed-bheduli & Herb, cl & wd, fe & All beats & - & Kamrul 123 (JUH) \\
\hline $\begin{array}{l}\text { Miliusa velutina (Dunal) Hook.f. \& } \\
\text { Thomson }\end{array}$ & Gandhi gajari & Tree, $\mathrm{m}$ & wd & Ad & Fr & Kamrul $1523(\mathrm{JUH})$ \\
\hline Polyalthia longifolia (Sonn.) Thwaites & Debdaru & Tree, 1 & $\mathrm{ml}$ & $\mathrm{Ka}$ & $\begin{array}{l}\mathrm{O}, \mathrm{T}, \\
\mathrm{M}\end{array}$ & Kamrul 2127 (JUH) \\
\hline $\begin{array}{l}\text { MYRISTICACEAE R.Br. } \\
\text { Knema cinerea Warb. } \\
\text { LAURACEAE Juss }\end{array}$ & Mota pasuti & Tree, $\mathrm{m}$ & wd & Ad & - & Kamrul $1574(J U H)$ \\
\hline $\begin{array}{l}\text { Actinodaphne gullavara (Buch.-Ham. ex } \\
\text { Nees) M.R. Almeida. }\end{array}$ & Modon mosta & Tree, $\mathrm{m}$ & hs & All beats & - & Kamrul 647 (JUH) \\
\hline $\begin{array}{l}\text { Cinnamomum tamala (Buch.-Ham.) T. } \\
\text { Nees \& Nees }\end{array}$ & Tejpata & Tree, $\mathrm{m}$ & $\mathrm{sj}$ & All beats & Sp, M & I Kamrul 1285 (JUH) \\
\hline Dehaasia kurzii Kingex Hook.f. * & Modon-mosto & Tree, $\mathrm{s}$ & hs & Ad & - & Kamrul $1022(\mathrm{JUH})$ \\
\hline Litsea monopetala (Roxb.) Pers. & Baro kukurchita & Tree, $\mathrm{s}$ & wd & All beats & $\begin{array}{l}\mathrm{M}, \mathrm{Fr}, \\
\mathrm{T}\end{array}$ & Kamrul 1445 (JUH) \\
\hline $\begin{array}{l}\text { Ocotea lancifolia (Schott) Mez. } \\
\text { CHLORANTHACEAE R.Br. ex Sims }\end{array}$ & Dulia & Tree, $\mathrm{s}$ & wd & Ad & $\mathrm{T}$ & Kamrul 298 (JUH) \\
\hline $\begin{array}{l}\text { Chloranthus erectus (Buch.-Ham.) } \\
\text { Verdc. }\end{array}$ & Rantas & Shrub & $\mathrm{fv}$ & Ad & $\mathrm{O}, \mathrm{M}$ & Kamrul 807 (JUH) \\
\hline PIPERACEAE Giseke & & & & & & \\
\hline Peperomia pellucida (L.) Kunth & Pithapata & Herb, e & $\mathrm{ml}$ & Ad & M & Kamrul $316(\mathrm{JUH})$ \\
\hline Piper betle $\mathrm{L}$. & Pan & Herb, cl & $\mathrm{ml}$ & Ad & M & Kamrul 11 (JUH) \\
\hline P. longum $\mathrm{L}$. & Pipul & Herb, cl & wd & Ad & M & Kamrul 2058 (JUH) \\
\hline P. nigrum $\mathrm{L}$. & Gol morich & Herb, cl & wd & Ad & M & Kamrul 723 (JUH) \\
\hline $\begin{array}{l}\text { P. sylvaticum Roxb. } \\
\text { ARISTOLOCHIACEAE Juss. }\end{array}$ & Ban pan & Herb, cr & $\mathrm{sj}, \mathrm{fe}$ & $\mathrm{Ad}, \mathrm{Ku}$ & M & Kamrul 83 (JUH) \\
\hline $\begin{array}{l}\text { Aristolochia tagala Cham. } \\
\text { SCHISANDRACEAE Blume }\end{array}$ & Iswararmul & Herb, cl & wd, hs & Ad & - & Kamrul 2139 (JUH) \\
\hline $\begin{array}{l}\text { Kadsura heteroclita (Roxb.) Craib } \\
\text { NYMPHAEACEAE Salisb. }\end{array}$ & Kadsuta & Shrub & wd & Ad & M & Kamrul 1432 (JUH) \\
\hline Nymphaea nouchali Burm. f. & Nilshapla & Herb, aq & $\mathrm{ml}$ & Ad & M & Kamrul $2233(\mathrm{JUH})$ \\
\hline $\begin{array}{l}\text { N. rubra Roxb. ex Andrews } \\
\text { CERATOPHYLLACEAE Grav }\end{array}$ & Lal shapla & Herb, aq & $\mathrm{ml}$ & Ad & $\mathrm{V}$ & Kamrul $2236(\mathrm{JUH})$ \\
\hline $\begin{array}{l}\text { CERATOPHYLLACEAE Gray } \\
\text { Ceratophyllum demersum L. }\end{array}$ & Sheola & Herb, aq & $\mathrm{ml}$ & Ad & M & Kamrul 2237 (JUH) \\
\hline RANUNCULACEAE Juss. & & & & & & \\
\hline $\begin{array}{l}\text { Nigella sativa } \mathrm{L} \text {. } \\
\text { MENISPERMACEAE Juss. }\end{array}$ & Kala-jeera & Herb, e & $\mathrm{ml}(\mathrm{cu})$ & $\mathrm{Ka}$ & M & Kamrul 2234 (JUH) \\
\hline Cissampelos pareira $\mathrm{L}$. & Akanadi & Herb, vi & wd & All beats & - & Kamrul $512(\mathrm{JUH})$ \\
\hline Cyclea barbata Miers & Patalpur & Herb, cl & wd & All beats & $\mathrm{M}$ & Kamrul $299(\mathrm{JUH})$ \\
\hline
\end{tabular}


Table 1 Contd.

\begin{tabular}{|c|c|c|c|c|c|c|}
\hline Scientific name & Bangla name & Habit & Habitat & Distrib. & Uses & RSE \\
\hline Diploclisia glaucescens (Blume) Diels & Sonatola & Herb, vi & wd & Ad & $\mathrm{M}$ & Kamrul 1295 (JUH) \\
\hline Pericampylus glaucus (Lam.) Merr. & Goria lata & Herb, cl & $\mathrm{fe}$ & $\mathrm{Ad}, \mathrm{Ku}$ & $\mathrm{Du}$ & Kamrul 1209 (JUH) \\
\hline $\begin{array}{l}\text { Pycnarrhena planiflora Miers ex Hook. } \\
\text { f. \& Thomson * }\end{array}$ & Henalora & Shrub & $\mathrm{ml}$ & $\mathrm{Ad}, \mathrm{Ku}$ & - & Kamrul 505 (JUH) \\
\hline Stephania japonica (Thunb.) Miers & Nimukha & Herb, cl & $\mathrm{Sj}, \mathrm{ml}$ & All beats & M & Kamrul 317 (JUH) \\
\hline $\begin{array}{l}\text { Tinospora sinensis (Lour.) Merr. } \\
\text { SABIACEAE Blume }\end{array}$ & Padma gulancha & Shrub, cl & $\mathrm{rb}, \mathrm{sj}$ & All beats & - & Kamrul 82 (JUH) \\
\hline Meliosma pinnata (Roxb.) Maxim. & Bativa & Tree & wd & Ad & $\mathrm{T}, \mathrm{Fr}$ & $\begin{array}{l}\text { S.N.Uddin N5025 } \\
\text { (DACB) }\end{array}$ \\
\hline M. simplicifolia (Roxb.) Walp. & Dibru & Tree & wd & Ad & $\mathrm{T}$ & Kamrul 1433 (JUH) \\
\hline Sabia lanceolata Colebr. & Sajba lat & Herb, cl & wd & All beats & $\mathrm{T}$ & Kamrul 1157 (JUH) \\
\hline $\begin{array}{l}\text { S. limoniacea Wall. ex Hook.f. \& Thomson } \\
\text { ULMACEAE Mirb. }\end{array}$ & Limo soobja & Herb, cl & wd & All beats & - & Kamrul 134 (JUH) \\
\hline Trema orientalis (L.) Blume & Banjiga & Tree, $\mathrm{m}$ & $\mathrm{rb}$ & $\mathrm{Ad}, \mathrm{Ku}$ & $\begin{array}{l}\text { Fw, } \\
\text { Fd }\end{array}$ & Kamrul $312(\mathrm{JUH})$ \\
\hline MORACEAE Gaudich. & & & & & & \\
\hline Artocarpus chama Buch.-Ham. & Chapalish & Tree, 1 & wd & All beats & $\mathrm{T}, \mathrm{Fr}$ & Kamrul $2174(\mathrm{JUH})$ \\
\hline A. heterophyllus Lam. & Kanthal & Tree, 1 & $\mathrm{ml}$ & Ad, Ka & $\mathrm{T}, \mathrm{Fr}$ & Kamrul 2037 (JUH) \\
\hline A. lacucha Buch.-Ham. & Deua & Tree, $\mathrm{m}$ & wd & Ad & $\begin{array}{l}\mathrm{Fr}, \mathrm{T}, \\
\mathrm{M}\end{array}$ & Kamrul 2070 (JUH) \\
\hline Ficus benghalensis $\mathrm{L}$. & Bot & Tree, 1 & $\mathrm{ml}$ & Ad & $\mathrm{O}, \mathrm{M}$ & Kamrul 2235 (JUH) \\
\hline F. benjamina $\mathrm{L}$. & Pakur & Tree, 1 & fe & $\mathrm{Ad}, \mathrm{Ku}$ & $\mathrm{O}, \mathrm{Fw}$ & Kamrul 841 (JUH) \\
\hline F. elastic Roxb. ex Hornem. & Rubber gach & Tree, $\mathrm{s}$ & $\mathrm{ml}$ & Ad & $\mathrm{O}$ & Kamrul 1458 (JUH) \\
\hline F. heterophylla L.f. & Bhui dumur & Shrub & $\mathrm{rb}$ & All beats & M & Kamrul 440 (JUH) \\
\hline F. hirta Vahl & Dadhuri & Shrub & fe & Ad & $\mathrm{Fd}$ & Kamrul 138 (JUH) \\
\hline F. hispida L.f. & Kakdumur & Shrub & $\mathrm{ml}$ & $\mathrm{Ad}$ & $\mathrm{Fr}, \mathrm{M}$ & Kamrul 303 (JUH) \\
\hline F. pumila $\mathrm{L}$. & Lata dumur & Herb, c & wd & Ad & $\mathrm{O}$ & Kamrul 2179 (JUH) \\
\hline F. racemosa $\mathrm{L}$. & Jagyadumur & Tree, $\mathrm{s}$ & $\mathrm{ml}$ & Ad & M & Kamrul 49 (JUH) \\
\hline F. religiosa $\mathrm{L}$. & Ashwath & Tree, 1 & $\mathrm{ml}$ & Ad & $\begin{array}{l}\mathrm{O}, \mathrm{Fr}, \\
\mathrm{M}\end{array}$ & Kamrul 861 (JUH) \\
\hline F. sagittata Vahl & Karat-bot & Herb, e & wd & $\mathrm{Ad}, \mathrm{Ku}$ & - & Kamrul 1368 (JUH) \\
\hline F. semicordata Buch.-Ham. ex Sm. & Sadimadi dumur & Tree, $\mathrm{s}$ & wd & $\mathrm{Ad}, \mathrm{Ku}$ & - & Kamrul 806 (JUH) \\
\hline F. variegata Blume & Bichitrabat & Tree, $\mathrm{s}$ & fv & Ad & Fw & Kamrul 843 (JUH) \\
\hline $\begin{array}{l}\text { Streblus asper Lour. } \\
\text { CECROPIACEAE C.C. Berg. }\end{array}$ & Sheora & Tree, $\mathrm{s}$ & $\mathrm{rb}$ & All beats & - & Kamrul $2180(\mathrm{JUH})$ \\
\hline $\begin{array}{l}\text { Poikilospermum suaveolens (Blume) } \\
\text { Merr. }\end{array}$ & Dolia sat & Tree, $\mathrm{m}$ & wd & Ad & - & Kamrul 157 (JUH) \\
\hline URTICACEAE Juss. & & & & & & \\
\hline Boehmeria glomerulifera Miq. & Borthurthuri & Shrub & wd & All beats & - & Kamrul 187 (JUH) \\
\hline B. macrophylla Hornem. & Ulichara & Shrub & wd & Ad & - & Kamrul 285 (JUH) \\
\hline Dendrocnide sinuata (Blume) Chew & Chutra & Shrub & wd & $\mathrm{Ad}$ & $\begin{array}{l}\mathrm{Fb}, \\
\mathrm{Fw}, \\
\mathrm{M}\end{array}$ & Kamrul 172 (JUH) \\
\hline Elatostema clarkei Hook. f. & Clarkejhara & Herb, e & wd & Ad, Ka & & Kamrul 820 (JUH) \\
\hline Laportea interrupta (L.) Chew & Lal bichuti & Herb, e & $\mathrm{ml}$ & All beats & M & Kamrul 2201 (JUH) \\
\hline Oreocnide integrifolia (Gaudich.) Miq. & Horhutta & Tree, $\mathrm{m}$ & wd & All beats & $\mathrm{Fb}$ & Kamrul 183 (JUH) \\
\hline Pouzolzia zeylanica (L.) Benn. & Kullaruki & Herb, pr & $\mathrm{ml}, \mathrm{gl}$ & Ad & M & Kamrul 837 (JUH) \\
\hline Pilea glaberrima (Blume) Blume & Glabrum & Shrub & wd & All beats & - & Kamrul 404 (JUH) \\
\hline Sarcochlamys pulcherrima Gaudich. & Marich & Tree, $\mathrm{m}$ & wd & Ad & Fw & Kamrul $1356(\mathrm{JUH})$ \\
\hline
\end{tabular}


Table 1 Contd.

\begin{tabular}{|c|c|c|c|c|c|c|}
\hline Scientific name & Bangla name & Habit & Habitat & Distrib. & Uses & RSE \\
\hline $\begin{array}{l}\text { JUGLANDACEAE DC. } \text { ex Perleb } \\
\text { Engelhardia spicata Lesch. ex Blume } \\
\text { FAGACEAE Dumort. }\end{array}$ & Jhumka bhadi & Tree, 1 & hs, fv & All beats & Sw & Kamrul 1457 (JUH) \\
\hline $\begin{array}{l}\text { Quercus obtusata Bonpl. } \\
\text { NYCTAGINACEAE Juss. }\end{array}$ & Batna & Tree, $\mathrm{m}$ & hs & Ad & - & Kamrul $1228(\mathrm{JUH})$ \\
\hline Boerhavia defusa $\mathrm{L}$. & Punarnava & Herb, pr & $\mathrm{ml}$ & All beats & M & Kamrul $2183(\mathrm{JUH})$ \\
\hline $\begin{array}{l}\text { Bougainvillea spectabilis Willd. } \\
\text { CHENOPODIACEAE Vent. }\end{array}$ & Bagan bilash & Shrub, c & $\mathrm{ml}$ & Ad, Ka & $\mathrm{O}$ & Kamrul 1147 (JUH) \\
\hline $\begin{array}{l}\text { Chenopodium album L. } \\
\text { AMARANTHACEAE Juss. }\end{array}$ & Bathua shak & Herb, cr & $\mathrm{ml}$ & Ad & M & Kamrul 1241 (JUH) \\
\hline Achyranthes aspera $\mathrm{L}$. & Apang & Herb, e & fe & All beats & M & Kamrul 2128 (JUH) \\
\hline Alternanthera paronychioides A.St.-Hil. & Jhuli khata & Herb, a & $\mathrm{ml}$ & Ad & M & Kamrul $2100(\mathrm{JUH})$ \\
\hline A. philoxeroides (Mart.) Griseb. & Henchi & Herb, a & $\mathrm{ml}$ & Ad & $\mathrm{V}$ & Kamrul 2137 (JUH) \\
\hline A. sessilis (L.) R.Br. ex DC. & Malancha & Herb, a & $\mathrm{ml}$ & Ad & $\mathrm{V}$ & Kamrul $2143(\mathrm{JUH})$ \\
\hline Amaranthus spinosus $\mathrm{L}$. & Kantanotey & Herb, e & $\mathrm{ml}$ & All beats & $\mathrm{V}, \mathrm{M}$ & Kamrul 282 (JUH) \\
\hline A. viridis $\mathrm{L}$. & Notey shak & Herb, e & $\mathrm{ml}$ & Ad & $\mathrm{V}$ & Kamrul $1639(\mathrm{JUH})$ \\
\hline $\begin{array}{l}\text { Cyathula prostrata (L.) Blume } \\
\text { POLYGONACEAE Juss. }\end{array}$ & Shyontula & Herb, pr & $\mathrm{fv}$ & All beats & M & Kamrul 886 (JUH) \\
\hline Persicaria hydropiper (L.) Delarbre & Biskatali & Herb, e & wd & All beats & - & Kamrul 38 (JUH) \\
\hline Polygonum effusum Meisn. & Raniphul & Herb, e & $\mathrm{ml}, \mathrm{gl}$ & $\mathrm{Ad}, \mathrm{Ku}$ & M & Kamrul $1716(\mathrm{JUH})$ \\
\hline P. lapathifolium $\mathrm{L}$. & Panibishkatali & Herb, e & wd & $\mathrm{Ad}, \mathrm{Ku}$ & M & Kamrul 903 (JUH) \\
\hline $\begin{array}{l}\text { Rumex maritimus L. } \\
\text { DILLENIACEAE Salisb. }\end{array}$ & Bon-palang & Herb, e & $\mathrm{wd}, \mathrm{rb}$ & $\mathrm{Ad}$ & M & Kamrul $153(\mathrm{JUH})$ \\
\hline Dillenia indica $\mathrm{L}$. & Chalta & Tree, $\mathrm{s}$ & $\mathrm{ml}$ & All beats & $\mathrm{Fr}, \mathrm{M}$ & Kamrul 669 (JUH) \\
\hline D. pentagyna Roxb. & Ban chalta & Tree, s & hs & Ad & $\mathrm{T}, \mathrm{M}$ & Kamrul 1453 (JUH) \\
\hline $\begin{array}{l}\text { Tetracera sarmentosa }(\mathrm{L} .) \text { Vahl } \\
\text { DIPTEROCARPACEAE Blume }\end{array}$ & Lata chalta & Herb, cr & wd & All beats & - & Kamrul 804 (JUH) \\
\hline Dipterocarpus turbinatus Gaertn. & Kali garjan & Tree, 1 & hs & Ad & $\mathrm{T}$ & Kamrul $2156(\mathrm{JUH})$ \\
\hline Hopea odorata Roxb. & Telsur & Tree, 1 & wd & Ad & $\mathrm{T}$ & Kamrul 2158 (JUH) \\
\hline $\begin{array}{l}\text { Shorea robusta Gaertn. } \\
\text { THEACEAE Mirb. }\end{array}$ & Sal & Tree, 1 & ht & $\mathrm{Ad}, \mathrm{Ka}$ & $\mathrm{T}$ & Kamrul 1175 (JUH) \\
\hline Eurya acuminata DC. & Sagoler bori & Shrub & wd & Ad & Fw & $\begin{array}{l}\text { S.N.Uddin N4813 } \\
\text { (DACB) }\end{array}$ \\
\hline $\begin{array}{l}\text { Schima wallichii (DC.) Korth. } \\
\text { ACTINIDIACEAE Gilg \& Werderm. }\end{array}$ & Bonak & Tree, $\mathrm{s}$ & ht, hs & All beats & $\mathrm{T}$ & Kamrul 551 (JUH) \\
\hline $\begin{array}{l}\text { Saurauia roxburghii Wall. } \\
\text { CLUSIACEAE Lindl. }\end{array}$ & Dalup & Tree, $\mathrm{m}$ & wd & Ad & $\mathrm{Fr}, \mathrm{Co}$ & Kamrul 534 (JUH) \\
\hline Garcinia cowa Roxb. ex Choisy & Cowa & Tree, $\mathrm{s}$ & wd & Ad & Fr & Kamrul 1075 (JUH) \\
\hline $\begin{array}{l}\text { Mesua ferrea } \text { L. } \\
\text { ELAEOCARPACEAE Juss. }\end{array}$ & Nagessawar & Tree, $s$ & wd & $\mathrm{Ad}$ & M & Kamrul 2151 (JUH) \\
\hline $\begin{array}{l}\text { Elaeocarpus petiolatus (Jack) Wall. * } \\
\text { TILIACEAE Juss. }\end{array}$ & Petipai & Tree, $\mathrm{m}$ & wd & All beats & M & Kamrul $1246(\mathrm{JUH})$ \\
\hline Corchorus aestuans L. & Titapat & Herb, e & $\mathrm{ml}$ & $\mathrm{Ka}$ & M & Kamrul $2238(\mathrm{JUH})$ \\
\hline Grewia nervosa (Lour.) Panigrahi & Pichandi & Tree, $\mathrm{s}$ & sj & All beats & M & Kamrul 1519 (JUH) \\
\hline G. serrrulata DC. & Pichandi & Shrub & sj & Ad & M & Kamrul 560 (JUH) \\
\hline G. tiliifolia Vahl & Jonli pholsa & Tree, $\mathrm{s}$ & sj & Ad & & Kamrul 784 (JUH) \\
\hline Triumfetta rhomboidea Jacq. & Bon okua & Herb, e & $\mathrm{gl}, \mathrm{ml}$ & All beats & M & Kamrul $98(J U H)$ \\
\hline T. pilosa Roth & Plofetta & Herb, e & $\mathrm{sj}$ & All beats & & Kamrul $1873(\mathrm{JUH})$ \\
\hline
\end{tabular}


Table 1 Contd.

\begin{tabular}{|c|c|c|c|c|c|c|}
\hline Scientific name & Bangla name & Habit & Habitat & Distrib. & Uses & RSE \\
\hline \multicolumn{7}{|l|}{ STERCULIACEAE Vent. } \\
\hline Abroma augusta (L.) L.f. & Ulatkambal & Shrub & sj & Ad & $\mathrm{M}, \mathrm{Fb}$ & Kamrul $2199(\mathrm{JUH})$ \\
\hline Byttneria aspera Colebr. ex Wall. & - & Liana & $\mathrm{e}$ & Ad & - & Kamrul 743 (JUH) \\
\hline B. pilosa Roxb. & Harjora lata & Liana & $\mathrm{e}$ & All beats & M & Kamrul $128(\mathrm{JUH})$ \\
\hline Melochia corchorifolia $\mathrm{L}$. & Tiki-okua & Herb, e & $f v$ & All beats & M & Kamrul 382 (JUH) \\
\hline Pterosprmum acerifolium (L.) Willd. & Kanackchampa & Tree, $\mathrm{m}$ & wd & All beats & $\begin{array}{l}\mathrm{O}, \mathrm{M}, \\
\mathrm{I}\end{array}$ & Kamrul 1896 (JUH) \\
\hline $\begin{array}{l}\text { Sterculia villosa Roxb. } \\
\text { BOMBACACEAE Kunth }\end{array}$ & Udal & Tree, $\mathrm{m}$ & hs & All beats & $\mathrm{Fr}$ & Kamrul $1278(\mathrm{JUH})$ \\
\hline Bombax ceiba $\mathrm{L}$. & Simul & Tree, 1 & wd & Ad & $\begin{array}{l}\mathrm{Sw} \\
\mathrm{Fb}\end{array}$ & Kamrul $190(\mathrm{JUH})$ \\
\hline $\begin{array}{l}\text { MALVACEAE Juss. } \\
\text { Abelmoschus moschatus Medik. }\end{array}$ & Mushak-dana & Herb, e & $\mathrm{ml}$ & All beats & $\begin{array}{l}\mathrm{M}, \mathrm{V} \\
\mathrm{O}\end{array}$ & Kamrul $410(\mathrm{JUH})$ \\
\hline Abutilon indicum (L.) Sweet & Petari & Herb, e & $\mathrm{ml}, \mathrm{gl}$ & All beats & $\mathrm{M}, \mathrm{Fb}$ & Kamrul $1710(\mathrm{JUH})$ \\
\hline Hibiscus rosa-sinensis $\mathrm{L}$. & Jaba & Shrub, e & $\mathrm{ml}$ & Ad & $\mathrm{O}$ & Kamrul 985 (JUH) \\
\hline H. macrophyllus Roxb. ex Hornem. & Udal & Tree, $\mathrm{m}$ & wd & All beats & & Kamrul 1771 (JUH) \\
\hline H. surattensis $\mathrm{L}$. & Ram bhindi & Shrub & hs & Ad & $\mathrm{M}$ & Kamrul 1601 (JUH) \\
\hline Malvaviscus arboreus Cav. & Marich jaba & Shrub & $\mathrm{ml}$ & Ad & $\mathrm{O}$ & Kamrul $2171(\mathrm{JUH})$ \\
\hline Sida acuta Burm. f. & Kureta & Herb, e & $\mathrm{rs}, \mathrm{ml}$ & All beats & M & Kamrul 1009 (JUH) \\
\hline S. cordata (Burm. f.) Borss. & Jumka & Herb, e & $\mathrm{ml}$ & All beats & - & Kamrul 335 (JUH) \\
\hline S. rhombifolia $\mathrm{L}$. & Lal berela & Herb, e & $\mathrm{ml}, \mathrm{rs}$ & $\mathrm{Ad}, \mathrm{Ka}$ & M & Kamrul 388 (JUH) \\
\hline $\begin{array}{l}\text { Urena lobata } \text { L. } \\
\text { LECYTHIDACEAE A. Rich. }\end{array}$ & Banghagra & Shrub & $\mathrm{rs}, \mathrm{ml}$ & All beats & M & Kamrul 685 (JUH) \\
\hline $\begin{array}{l}\text { Barringtonia acutangula (L.) Gaertn. } \\
\text { FLACOURTIACEAE Rich. } e x \text { DC. }\end{array}$ & Hijol & Tree, $\mathrm{m}$ & $\mathrm{ml}$ & Ad & M & Kamrul 2133 (JUH) \\
\hline Flacourtia indica (Burm. f.) Merr. & Bauchi & Shrub & sj & $\mathrm{Ad}, \mathrm{Ka}$ & $\begin{array}{l}\text { M, } \\
\text { Fr,T }\end{array}$ & Kamrul $1624(\mathrm{JUH})$ \\
\hline $\begin{array}{l}\text { F. jangomas (Lour.) Raeusch. } \\
\text { PASSILORACEAE Juss. ex Roussel }\end{array}$ & Lukluki & Tree, $\mathrm{s}$ & wd & All beats & $\mathrm{Fr}$ & Kamrul 1332 (JUH) \\
\hline $\begin{array}{l}\text { Adenia trilobata (Roxb.) Engl. } \\
\text { CARICACEAE Dumort. }\end{array}$ & Akandaphal & Herb, cl & wd, sj & $\mathrm{Ad}, \mathrm{Ku}$ & M & Kamrul 1324 (JUH) \\
\hline $\begin{array}{l}\text { Carica papaya } \mathrm{L} . \\
\text { CUCURBITACEAE Juss. }\end{array}$ & Pape & Tree, $\mathrm{s}$ & $\mathrm{ml}$ & Ad & $\mathrm{Fr}, \mathrm{V}$ & Kamrul $2259(\mathrm{JUH})$ \\
\hline Coccinia grandis (L.) Voigt & Telakucha & Herb, cl & $\mathrm{gl}, \mathrm{rs}$ & $\mathrm{Ad}, \mathrm{Ku}$ & M & Kamrul 2055 (JUH) \\
\hline $\begin{array}{l}\text { Gynostemma pentaphyllum (Thunb.) } \\
\text { Makino }\end{array}$ & Gymnopada & Herb, cr & $\mathrm{fv}$ & Ad & M & Kamrul 466 (JUH) \\
\hline Hodgsonia macrocarpa (Blume) Cogn.* & Makal maco & Liana & $\mathrm{sb}$ & Ad & M & Kamrul $2153(\mathrm{JUH})$ \\
\hline Luffa cylindrica (L.) M. Roem. & Dhundal & Herb, cl & ht & $\mathrm{Ku}$ & $\mathrm{V}, \mathrm{M}$ & Kamrul 1569 (JUH) \\
\hline Momordica charantia $\mathrm{L}$. & Karola & Herb, cl & $\mathrm{ml}(\mathrm{cl})$ & Ad & $\mathrm{V}$ & Kamrul $2053(\mathrm{JUH})$ \\
\hline M. dioica Roxb. ex Willd. & Ghee korolla & Herb, cl & $\mathrm{ml}(\mathrm{cl})$ & $\mathrm{Ad}$ & $\mathrm{V}, \mathrm{M}$ & Kamrul 2054 (JUH) \\
\hline Mukia maderaspatana (L.) M. Roem. & Bilari & Herb, cl & $\mathrm{gl}$, wd & Ad & M & Kamrul 1425 (JUH) \\
\hline Thladiantha cordifolia (Blume) Cogn. & - & Herb, cl & sj & Ad & - & Kamrul 1423 (JUH) \\
\hline $\begin{array}{l}\text { Trichosanthes tricuspidata Lour. } \\
\text { BEGONIACEAE C. Agardh }\end{array}$ & Makal & Herb, cl & wd & All beats & - & Kamrul 1438 (JUH) \\
\hline Begonia annulata K. Koch. & Gonibata & Herb, e & sj & Ad & $\mathrm{O}, \mathrm{V}$ & Kamrul 1908 (JUH) \\
\hline $\begin{array}{l}\text { B. roxburghii (Miq.) A.DC. } \\
\text { CAPPARACEAE Juss. }\end{array}$ & Gonorakto & Herb, e & sj & All beats & $\mathrm{O}, \mathrm{V}$ & Kamrul 825 (JUH) \\
\hline Stixis suaveolens (Roxb.) Pierre & Madhumaloti & Herb, cl & $\mathrm{wd}, \mathrm{rb}$ & All beats & $\mathrm{Fr}$ & Kamrul 05 (JUH) \\
\hline
\end{tabular}


Table 1 Contd.

\begin{tabular}{|c|c|c|c|c|c|c|}
\hline Scientific name & Bangla name & Habit & Habitat & Distrib. & Uses & RSE \\
\hline \multicolumn{7}{|l|}{ BRASSICACEAE Burnett } \\
\hline Rorippa indica (L.) Hiern & Bansarisha & Herb, e & wd & $\mathrm{Ka}$ & - & Kamrul 2240 (JUH) \\
\hline \multicolumn{7}{|l|}{ SAPOTACEAE Juss. } \\
\hline $\begin{array}{l}\text { Madhuca longifolia (J. Koenig ex L.) J.F. } \\
\text { Macbr. }\end{array}$ & . Mohua & Tree, $\mathrm{m}$ & $\mathrm{ml},(\mathrm{pl})$ & Ad & M & Kamrul $2190(\mathrm{JUH})$ \\
\hline $\begin{array}{l}\text { Mimusops elengi } \mathrm{L} . \\
\text { EBENACEAE Gürke }\end{array}$ & \multicolumn{6}{|c|}{ EBENACEAE Gürke } \\
\hline $\begin{array}{l}\text { Diospyros malabarica (Desr.) Kostel. } \\
\text { STYRACACEAE DC. \& Spreng. }\end{array}$ & Deshi gab & Tree, 1 & $\mathrm{ml}$ & Ad & M & Kamrul 2241(JUH) \\
\hline \multicolumn{7}{|l|}{ SYMPLOCACEAE Desf. } \\
\hline $\begin{array}{l}\text { Symplocos macrophylla Wall. ex A.DC.* } \\
\text { PRIMULACEAE Batsch ex Borkh. }\end{array}$ & Barabahuri & Tree, $\mathrm{m}$ & hs & Ad & - & Kamrul $536(\mathrm{JUH})$ \\
\hline Ardisia sanguinolenta Blume & - & Shrub & wd & Ad, Ka & M & Kamrul 1121 (JUH) \\
\hline Embelia ribes Burm. $\mathrm{f}$. & Bakul lata & Shrub & $\mathrm{fv}$ & Ad & $\mathrm{M}, \mathrm{Fr}$ & Kamrul 1252 (JUH) \\
\hline Hymenandra wallichii A. DC. & Bhau jawa & Shrub & wd & $\mathrm{Ad}, \mathrm{Ku}$ & - & Kamrul 301 (JUH) \\
\hline Maesa bengalensis Mez. & Banglauni & Tree, $\mathrm{s}$ & wd & Ad & - & Kamrul 1411 (JUH) \\
\hline M. chisia Buch.-Ham. ex D. Don & Gangu lata & Shrub & wd & All beats & - & Kamrul 1660 (JUH) \\
\hline M. indica (Roxb.) A. DC. & Sesu & Shrub & wd & All beats & $\mathrm{M}, \mathrm{V}$ & Kamrul 966 (JUH) \\
\hline M. ramentacea (Roxb.) A. DC. & Noa-maricha & Shrub & wd & All beats & M & Kamrul 544 (JUH) \\
\hline CRASSULACEAE J.St.-Hil. & & & & & & \\
\hline $\begin{array}{l}\text { Bryophyllum pinnatum (Lam.) Oken } \\
\text { ROSACEAE Juss. }\end{array}$ & Pathorkuchi & Herb, cr & $\mathrm{ml}$ & Ad & $\mathrm{O}, \mathrm{M}$ & Kamrul $2242(\mathrm{JUH})$ \\
\hline Prunus ceylanica Miq. & Ceylon cherry & Tree, $\mathrm{s}$ & sj & Ad & $\mathrm{O}, \mathrm{Sw}$ & Kamrul 1587 (JUH) \\
\hline Rubus hexagynus Roxb. & Hirachura & Shrub & hs & $\mathrm{Ad}$ & - & Kamrul $1328(J U H)$ \\
\hline Rosa chinensis Jacq & Kata golap & Shrub & $\mathrm{ml}$ & Ad & $\mathrm{O}$ & Kamrul 1106 (JUH) \\
\hline \multicolumn{7}{|l|}{ MIMOSACEAE R.Br. } \\
\hline Mimosa pudica L. & Lajjaboti & Shrub & $\mathrm{ml}, \mathrm{sj}$ & All beats & $\mathrm{M}$ & Kamrul 433 (JUH) \\
\hline Acacia auriculiformis A.Cunn. ex Benth. & Akashmoni & Tree, 1 & $\mathrm{ml}(\mathrm{pl})$ & All beats & $\mathrm{T}$ & Kamrul $1450(\mathrm{JUH})$ \\
\hline A. mangium Willd. & Mangium & Tree, 1 & $\mathrm{ml}(\mathrm{pl})$ & All beats & $\mathrm{T}$ & Kamrul $2112(\mathrm{JUH})$ \\
\hline \multicolumn{7}{|l|}{ CAESALPINIACEAE R.Br. } \\
\hline Cassia fistula $\mathrm{L}$. & Bandar lathi & Tree, $\mathrm{m}$ & wd & All beats & $\begin{array}{l}\text { M, St, } \\
\mathrm{T}\end{array}$ & Kamrul $1212(\mathrm{JUH})$ \\
\hline Caesalpinia bonduc (L.) Roxb. & Nata & Shrub, sc & sj & $\mathrm{Ka}$ & M & Kamrul $1223(\mathrm{JUH})$ \\
\hline C. enneaphyllum Roxb. & Nataine & Herb, e & $\mathrm{sj}$ & $\mathrm{Ka}$ & $\mathrm{O}$ & Kamrul 1014 (JUH) \\
\hline Delonix regia (Bojer ex Hook.) Raf. & Kuishnachura & Tree, $\mathrm{m}$ & $\mathrm{ml}(\mathrm{pl})$ & $\mathrm{Ka}$ & $\mathrm{O}, \mathrm{Sw}$ & Kamrul 2150 (JUH) \\
\hline Senna alata (L.) Roxb. & Dadmardan & Shrub & $\mathrm{ml}, \mathrm{rs}$ & $\mathrm{Ka}$ & M & Kamrul 734 (JUH) \\
\hline S. siamea (Lam.) H.S. Irwin \& Barneby & Minjiri & Tree, $\mathrm{s}$ & rs & All beats & $\mathrm{O}$ & Kamrul 561 (JUH) \\
\hline S. sophera (L.) Roxb. & Kalkashunda & Shrub & $\mathrm{ml}, \mathrm{sj}$ & $\mathrm{Ka}$ & M & Kamrul 1807 (JUH) \\
\hline S. tora (L.) Roxb. & Terasena & Herb, e & $\mathrm{ml}, \mathrm{rs}$ & All beats & M & Kamrul 375 (JUH) \\
\hline Tamarindus indica $\mathrm{L}$. & Tetul & Tree, 1 & $\mathrm{ml}(\mathrm{pl})$ & $\mathrm{Ka}$ & $\begin{array}{l}\mathrm{Fr}, \\
\mathrm{M}, \mathrm{T}\end{array}$ & Kamrul 580 (JUH) \\
\hline \multicolumn{7}{|l|}{ FABACEAE Lindl. } \\
\hline Cajanus scarabaeoides (L.) Thouars & Orhor & Shrub & hs & $\mathrm{Ka}$ & M & Kamrul 2134 (JUH) \\
\hline Dalbergia stipulacea Roxb. & Dadbari & Herb, e & wd & All beats & M & Kamrul $1111(\mathrm{JUH})$ \\
\hline $\begin{array}{l}\text { Dalhousiea bracteata (Roxb.) Graham } \\
\text { ex Benth.* }\end{array}$ & Gupuri & Shrub & $\mathrm{rb}, \mathrm{wd}$ & Ad & - & Kamrul $1586(\mathrm{JUH})$ \\
\hline Derris robusta (Roxb. ex DC.) Benth. & Korai & Tree, 1 & hs & All beats & - & Kamrul $1286(\mathrm{JUH})$ \\
\hline
\end{tabular}


Table 1 Contd.

\begin{tabular}{|c|c|c|c|c|c|c|}
\hline Scientific name & Bangla name & Habit & Habitat & Distrib. & Uses & RSE \\
\hline$\overline{\text { Desmodium gangeticum (L.) DC. }}$ & Chalani & Shrub & $\mathrm{rs}, \mathrm{ml}$ & All beats & M & Kamrul 500 (JUH) \\
\hline D. heterophyllum (Willd.) DC. & Bon motorsuti & Herb, pr & rs & All beats & M & Kamrul 1006 (JUH) \\
\hline D. heterocarpon (L.) DC. & Karpo modi & Shrub & $\mathrm{fe}$ & All beats & - & Kamrul 752 (JUH) \\
\hline D. laxiflorum DC. & Laximodi & Shrub & wd & All beats & M & Kamrul 839 (JUH) \\
\hline Tadehagi triquetrum (L.) H. Ohashi & Kanimanda & Shrub & hs & Ad & - & Kamrul 1153 (JUH) \\
\hline Erythrina variegata $\mathrm{L}$. & Bahari mander & Tree, $\mathrm{m}$ & $\mathrm{ml}$ & $\mathrm{Ad}$ & $\begin{array}{l}\mathrm{O}, \mathrm{T} \\
\mathrm{M}\end{array}$ & Kamrul 1461 (JUH) \\
\hline Flemingia involucrata Benth. & Vuluk phan & Shrub & wd & All beats & - & Kamrul 2115 (JUH) \\
\hline F. macrophylla (Willd.) Kuntze ex Merr. & Baro salpan & Shrub & wd & All beats & Dy & Kamrul 147 (JUH) \\
\hline F. strobilifera (L.) W.T. Aiton & Chingri pata & Shrub & $\mathrm{ml}$ & All beats & M & Kamrul 925 (JUH) \\
\hline Indigofera zollingeriana Miq. & Gerina nil & Shrub & fe & All beats & St & Kamrul 56 (JUH) \\
\hline Lathyrus sativus L. & Khesari & Herb, e & $\mathrm{ml}(\mathrm{cl})$ & Ad & $\mathrm{Fd}, \mathrm{Pu}$ & Kamrul 1708 (JUH) \\
\hline Millettia pachycarpa Benth. & Bish lata & Liana & hs & Ad & I & Kamrul $1388(\mathrm{JUH})$ \\
\hline Mucuna pruriens (L.) DC. & Alkushi & Herb, c & $\mathrm{sj}$ & Ad & $\mathrm{M}$ & Kamrul 392 (JUH) \\
\hline Pueraria phaseoloides (Roxb.) Benth. & Mugi kunch & Herb, e & sj & All beats & $\begin{array}{l}\mathrm{Fd}, \\
\mathrm{Gm}\end{array}$ & Kamrul 1091 (JUH) \\
\hline Spatholobus parviflorus (DC.) Kuntze & Pan lata & Herb, c & sj & Ad & $\mathrm{M}, \mathrm{Du}$ & Kamrul 1389 (JUH) \\
\hline Tephrosia candida (Roxb.) DC. & Bilakshani & Herb, e & rs & $\mathrm{Ad}$ & $\begin{array}{l}\mathrm{M}, \\
\mathrm{Gm}\end{array}$ & Kamrul 1034 (JUH) \\
\hline Uraria crinita (L.) Desv. ex DC. & Diangleja & Shrub & sj & Ad & M & Kamrul 424 (JUH) \\
\hline $\begin{array}{l}\text { Vigna mungo (L.) Hepper } \\
\text { SONNERATIACEAE Engl. }\end{array}$ & Maskalay & Herb, e & $\mathrm{ml}(\mathrm{cl})$ & Ad & $\mathrm{Pu}$ & Kamrul 1087 (JUH) \\
\hline $\begin{array}{l}\text { Duabanga grandiflora (Roxb. ex DC.) } \\
\text { Walp. }\end{array}$ & Bandorhola & Tree, 1 & $f v, r b$ & Ad & $\mathrm{T}$ & Kamrul 1481 (JUH) \\
\hline LYTHRACEAE J.St.-Hil. & & & & & & \\
\hline Ammannia multiflora Roxb. & Acidpata & Herb, e & fv & Ad & - & Kamrul $1858(\mathrm{JUH})$ \\
\hline Lagerstroemia speciosa (L.) Pers. & Jarul & Tree, $\mathrm{m}$ & $\mathrm{ml}$ & All beats & $\begin{array}{l}\mathrm{O}, \mathrm{T} \\
\mathrm{M}\end{array}$ & Kamrul 289 (JUH) \\
\hline Lawsonia inermis $\mathrm{L}$. & Mendi & Shrub & $\mathrm{ml}$ & $\mathrm{Ka}$ & Dy & Kamrul $1290(\mathrm{JUH})$ \\
\hline Rotala indica (Willd.) Koehne & Deshi ghurni & Herb, cr & $\mathrm{ml}$ & All beats & - & Kamrul 1140 (JUH) \\
\hline $\begin{array}{l}\text { R. rotundifolia (Buch.-Ham. ex Roxb.) } \\
\text { Koehne } \\
\text { THYMELAEACEAE Juss. }\end{array}$ & Dim ghurni & Herb, cr & $\mathrm{ml}$ & All beats & - & Kamrul 6(JUH) \\
\hline $\begin{array}{l}\text { Aquilaria agallocha } \text { Roxb. * } \\
\text { MYRTACEAE Juss. }\end{array}$ & Agar & Tree, $\mathrm{m}$ & $\mathrm{ml}$ & Ad, Ka & $\mathrm{Pe}$ & Kamrul $1144(\mathrm{JUH})$ \\
\hline $\begin{array}{l}\text { Corymbia citriodora (Hook.) K.D. Hill } \\
\& \text { L.A.S. Johnson }\end{array}$ & Eucalyptus & Tree, 1 & $\mathrm{ml}(\mathrm{pl})$ & $\mathrm{Ka}$ & $\mathrm{T}$ & Kamrul 2249 (JUH) \\
\hline Psidium guajava $\mathrm{L}$ & Peyara & Tree, $\mathrm{s}$ & $\mathrm{ml}$ & $\mathrm{Ad}, \mathrm{Ka}$ & $\mathrm{Fr}, \mathrm{M}$ & Kamrul 2095 (JUH) \\
\hline Syzygium cumini (L.) Skeels & Kalojam & Tree, 1 & $\mathrm{rs}, \mathrm{ml}$ & All beats & $\mathrm{Fr}, \mathrm{T}$ & Kamrul $1136(\mathrm{JUH})$ \\
\hline S. fruticosum DC. & Ban Jam & Tree, 1 & wd & $\mathrm{Ad}, \mathrm{Ku}$ & Fr, T & Kamrul 2096 (JUH) \\
\hline $\begin{array}{l}\text { S. grande (Wight) Walp. } \\
\text { ONAGRACEAE Juss. }\end{array}$ & Dhaki Jam & Tree, 1 & $\mathrm{fe}$ & $\mathrm{Ad}, \mathrm{Ku}$ & $\mathrm{Fr}, \mathrm{T}$ & Kamrul $1020(\mathrm{JUH})$ \\
\hline Ludwigia octovalvis (Jacq.) P.H.Raven & Bhuikura & Herb, e & $\mathrm{ml}$ & Ad & - & $\begin{array}{l}\text { S.N.Uddin N4779 } \\
\text { (DACB) }\end{array}$ \\
\hline $\begin{array}{l}\text { L. perennis L. } \\
\text { MELASTOMATACEAE Juss. }\end{array}$ & Amorkura & Herb, e & $\mathrm{ml}$ & All beats & - & Kamrul 144 (JUH) \\
\hline Melastoma malabathricum L. & Ban tejpata & Shrub & Wd, fe, $\mathrm{m}$ & 1 All beats & M & Kamrul 10 (JUH) \\
\hline Osbeckia nepalensis Hook.f. & Nepaligachi & Shrub & $\mathrm{Wd}, \mathrm{fe}$ & Ad & - & Kamrul $1828(\mathrm{JUH})$ \\
\hline
\end{tabular}


Table 1 Contd.

\begin{tabular}{|c|c|c|c|c|c|c|}
\hline Scientific name & Bangla name & Habit & Habitat & Distrib. & Uses & RSE \\
\hline \multicolumn{7}{|l|}{ COMBRETACEAE R. Br. } \\
\hline Combretum acuminatum Roxb. & Patyuni & Shrub & wd & All beats & - & Kamrul 2248 (JUH) \\
\hline C. roxburghii Spreng. & Kaligaichi & Herb, e & wd & Ad & - & Kamrul 1033 (JUH) \\
\hline C. wallichii DC. & Yunanlata & Herb, e & wd & Ad & - & Kamrul 178 (JUH) \\
\hline $\begin{array}{l}\text { Terminalia arjuna (Roxb. ex DC.) Wight } \\
\& \text { Arn. }\end{array}$ & Arjun & Tree, 1 & wd & $\mathrm{Ka}$ & M & Kamrul 566 (JUH) \\
\hline T. bellirica (Gaertn.) Roxb. & Bohera & Tree, 1 & rs & $\mathrm{Ka}$ & $\mathrm{M}$ & Kamrul $582(\mathrm{JUH})$ \\
\hline T. catappa $\mathrm{L}$ & Kathbadam & Tree, 1 & rs & $\mathrm{Ka}$ & M & Kamrul 2152 (JUH) \\
\hline T. chebula Retz. & Horitoki & Tree, 1 & wd & $\mathrm{Ka}$ & M & Kamrul 583 (JUH) \\
\hline RHIZOPHORACEAE Pers. & & & & & & \\
\hline $\begin{array}{l}\text { Carallia brachiata (Lour.) Merr. } \\
\text { OLACACEAE Juss. ex R. Br. }\end{array}$ & Rascow & Tree, $\mathrm{m}$ & wd & Ad & $\mathrm{T}, \mathrm{M}$ & Kamrul $860(\mathrm{JUH})$ \\
\hline $\begin{array}{l}\text { Olax acuminata Wall. ex Benth. } \\
\text { LORANTHACEAE Juss. }\end{array}$ & Capsul gach & Shrub & sj & All beats & - & Kamrul 349 (JUH) \\
\hline Helixanthera parasitica Lour. & Xanthric & Shrub & hs & Ad & - & Kamrul 1299 (JUH) \\
\hline $\begin{array}{l}\text { Macrosolen cochinchinensis (Lour.) } \\
\text { Tiegh. }\end{array}$ & Chota banda & Shrub & wd & Ad & - & Kamrul $1104(\mathrm{JUH})$ \\
\hline $\begin{array}{l}\text { Scurrula parasitica } \text { L. } \\
\text { CELASTRACEAE R.Br. }\end{array}$ & Pargacha & Shrub & hs & Ad & - & Kamrul 1088 (JUH) \\
\hline $\begin{array}{l}\text { Bhesa robusta (Roxb.) Ding Hou* } \\
\text { HIPPOCRATEACEAE Juss. }\end{array}$ & Madhu-phal & Tree, $\mathrm{m}$ & wd & Ad & $\mathrm{T}$ & Kamrul 1097 (JUH) \\
\hline $\begin{array}{l}\text { Salacia chinensis L. } \\
\text { AQUIFOLIACEAE Bercht. \& J. Pres1 }\end{array}$ & Vesa & Shrub, sc & wd & Ad & $\mathrm{M}, \mathrm{Fr}$ & Kamrul 1237 (JUH) \\
\hline $\begin{array}{l}\text { Ilex godajam (Colebr. ex Wall.) Wall. ex } \\
\text { Hook. f. }\end{array}$ & Raktim & Shrub & hs & Ad & $\mathrm{Fw}$ & Kamrul 1777 (JUH) \\
\hline EUPHORBIACEAE Juss. & & & & & & \\
\hline Acalypha indica $\mathrm{L}$. & Muktajhuri & Herb, e & $\mathrm{ml}$ & All beats & M & Kamrul 2159 (JUH) \\
\hline Actephila excelsa (Dalzell) Müll.-Arg. & Lalsa & Shrub & hs & $\mathrm{Ku}$ & $\mathrm{V}, \mathrm{M}$ & Kamrul 1577 (JUH) \\
\hline Alchornea tiliifolia (Benth.) Müll.-Arg. & Alkotil & Shrub & $\mathrm{sj}, \mathrm{fv}$ & Ad & $\mathrm{Fw}$ & Kamrul $2162(\mathrm{JUH})$ \\
\hline Antidesma acidum Retz. & Titij am & Shrub & wd & All beats & Fr & Kamrul 649 (JUH) \\
\hline A. ghaesembilla Gaertn. & Khudi jam & Tree, $\mathrm{s}$ & wd & Ad & M & Kamrul 921 (JUH) \\
\hline A. montanum Blume & Shial buka & Shrub & wd & All beats & $\mathrm{Fr}$ & Kamrul 366 (JUH) \\
\hline Aporosa wallichii Hook. f. & Kokua & Tree, $\mathrm{s}$ & wd & All beats & - & Kamrul 154 (JUH) \\
\hline Bischofia javanica Blume & Kainjal & Tree, $\mathrm{s}$ & fe & Ad & $\mathrm{T}, \mathrm{M}$ & Kamrul 1396 (JUH) \\
\hline Bridelia tomentosa Blume & Khoi & Shrub & $\mathrm{sj}$ & All beats & M & Kamrul 1103 (JUH) \\
\hline Baccaurea ramiflora Lour. & Latkan & Tree, $\mathrm{m}$ & wd & Ad & $\mathrm{Fr}, \mathrm{M}$ & Kamrul $1476(\mathrm{JUH})$ \\
\hline $\begin{array}{l}\text { Chaetocarpus castanocarpus (Roxb.) } \\
\text { Thwaites }\end{array}$ & Dhala kakua & Tree, $\mathrm{m}$ & wd & Ad & - & Kamrul 143 (JUH) \\
\hline Cnesmone javanica Blume & Chutra & Shrub & $\mathrm{Sj}$, ht & All beats & M & Kamrul 383 (JUH) \\
\hline Croton bonplandianus Baill. & Banmarich & Herb, e & $\mathrm{ml}, \mathrm{rs}$ & All beats & M & Kamrul 481 (JUH) \\
\hline $\begin{array}{l}\text { Baliospermum solanifolium (Burm.) } \\
\text { Suresh }\end{array}$ & Chuka & Shrub & $\mathrm{rb}$ & Ad & M & Kamrul 125 (JUH) \\
\hline Euphorbia hirta L. & Bara dudhia & Herb, pr & $\mathrm{ml}, \mathrm{rs}$ & All beats & M & Kamrul $2164(\mathrm{JUH})$ \\
\hline $\begin{array}{l}\text { Glochidion multiloculare (Rottler } e x \\
\text { Willd.) Voigt }\end{array}$ & Aniatori & Shrub & hs & All beats & $\mathrm{T}$ & Kamrul 32 (JUH) \\
\hline Macaranga indica Wight. & Gulle & Tree, $\mathrm{s}$ & wd & All beats & M & Kamrul 1231 (JUH) \\
\hline Phyllanthus emblica L. & Amloki & Tree, $\mathrm{m}$ & $\mathrm{sj}$ & $\mathrm{Ka}$ & $\mathrm{Fr}, \mathrm{M}$ & Kamrul 554 (JUH) \\
\hline P. niruri $\mathrm{L}$. & Bhuiamla & Herb, e & $\mathrm{ml}$ & Ad & M & Kamrul $760(\mathrm{JUH})$ \\
\hline P. reticulatus Poir. & Pankushi & Shrub & $\mathrm{sj}, \mathrm{rb}$ & All beats & M & Kamrul 1499 (JUH) \\
\hline
\end{tabular}


Table 1 Contd.

\begin{tabular}{|c|c|c|c|c|c|c|}
\hline Scientific name & Bangla name & Habit & Habitat & Distrib. & Uses & RSE \\
\hline P. urinaria $\mathrm{L}$. & Hazarmani & Herb, e & sj & Ad & M & Kamrul $79(\mathrm{JUH})$ \\
\hline Suregada multiflora (A.Juss.) Baill. & Ban naranga & Tree, s & wd & Ad & $\mathrm{T}$ & Kamrul 226 (JUH) \\
\hline Ricinus communis $\mathrm{L}$. & Bherenda & Shrub & wd & Ad & M & Kamrul 2167 (JUH) \\
\hline $\begin{array}{l}\text { Sauropus androgynus (L.) Merr. } \\
\text { RHAMNACEAE Juss. }\end{array}$ & Mithapotro & Shrub & wd & Ad & M & Kamrul 1397 (JUH) \\
\hline Gouania tiliifolia Lam. & Herjengota & Shrub & sj & $\mathrm{Ad}, \mathrm{Ku}$ & $\mathrm{V}$ & Kamrul $1901(\mathrm{JUH})$ \\
\hline Ziziphus mauritiana Lam. & Bol boroi & Tree, $\mathrm{s}$ & $\mathrm{ml}$ & All beats & M & Kamrul 571 (JUH) \\
\hline $\begin{array}{l}\text { Z. oenopolia (L.) Mill. } \\
\text { LEEACEAE Dumort. }\end{array}$ & Ban boroi & Shrub & sj & All beats & M & Kamrul 482 (JUH) \\
\hline Leea guineensis G. Don. & - & Shrub & hs & All beats & - & Kamrul 604 (JUH) \\
\hline $\begin{array}{l}\text { L. indica (Burm.f.) Merr. } \\
\text { VITACEAE Juss. }\end{array}$ & Kurkurjhibba & Shrub & $\mathrm{fv}$ & All beats & M & Kamrul 241 (JUH) \\
\hline Ampelocissus latifolia (Roxb.) Planch. & Gowalia lata & Herb, c & $\mathrm{rb}, \mathrm{sj}$ & Ad & - & Kamrul 2204 (JUH) \\
\hline Cissus javana DC. & Dukhu lata & Liana & wd & All beats & $\mathrm{O}$ & Kamrul 702 (JUH) \\
\hline C. adnata Roxb. & Bhatia lata & Liana & wd & All beats & M & Kamrul 639 (JUH); \\
\hline Cayratia japonica (Thunb.) Gagnep. & Japani goali lata & Herb, cl & $\mathrm{rb}$ & Ad & M & Kamrul 508 (JUH) \\
\hline C. trifolia $($ L.) Domin & Amal lata & Herb, cl & $\mathrm{rb}$ & All beats & M & Kamrul $1118(\mathrm{JUH})$ \\
\hline $\begin{array}{l}\text { Tetrastigma lanceolarium (Roxb.) } \\
\text { Planch. }\end{array}$ & - & Herb, e & ht & Ad & - & Kamrul $1589(\mathrm{JUH})$ \\
\hline $\begin{array}{l}\text { T. leucostaphylum (Dennst.) Alston } \\
\text { POLYGALACEAE Hoffeanns. \& Link }\end{array}$ & Horina lata & Shrub & wd & $\mathrm{Ad}, \mathrm{Ku}$ & - & Kamrul 739 (JUH) \\
\hline Polygala chinensis L. & Meradu & Herb, pr & hs, sj & $\mathrm{Ka}$ & M & Kamrul $1428(\mathrm{JUH})$ \\
\hline $\begin{array}{l}\text { P. erioptera DC. } \\
\text { STAPHYLEACEAE Martinov }\end{array}$ & Teradudhi & Herb, pr & $\mathrm{ml}$ & All beats & - & Kamrul $2188(\mathrm{JUH})$ \\
\hline Turpinia pomifera (Roxb.) DC. & Bhola & Tree & wd & Ad & $\begin{array}{l}\mathrm{Fd}, \\
\mathrm{Fw}\end{array}$ & Kamrul $1459(\mathrm{JUH})$ \\
\hline $\begin{array}{l}\text { SAPINDACEAE Juss. } \\
\text { Allophylus cobbe (L.) Raeusch. }\end{array}$ & Chita & Shrub & wd & All beats & - & Kamrul 719 (JUH) \\
\hline Lepisanthes senegalensis (Poir.) Leenh. & Chita & Shrub & wd & $\mathrm{Ad}, \mathrm{Ku}$ & - & Kamrul 1029 (JUH) \\
\hline $\begin{array}{l}\text { Litchi chinensis Sonn. } \\
\text { BURSERACEAE Kunth }\end{array}$ & Lichu & Tree, 1 & $\mathrm{ml}(\mathrm{pl})$ & Ad & $\mathrm{Fr}$ & Kamrul $2189(\mathrm{JUH})$ \\
\hline $\begin{array}{l}\text { Garuga pinnata } \text { Roxb. } \\
\text { ANACARDIACEAE } \mathrm{R} \text {. Br. }\end{array}$ & Paharijiga & Tree, $\mathrm{m}$ & wd & All beats & $\mathrm{T}, \mathrm{M}$ & Kamrul 780 (JUH) \\
\hline Anacardium occidentale $\mathrm{L}$. & Kaju. & Tree, $\mathrm{m}$ & rs & $\mathrm{Ka}$ & Fr & Kamrul $1201(\mathrm{JUH})$ \\
\hline Lannea coromandelica (Houtt.) Merr. & Jiga & Tree, $\mathrm{m}$ & rs & Ad & M & Kamrul 1451 (JUH) \\
\hline Mangifera indica $\mathrm{L}$. & Aam & Tree, 1 & $\mathrm{ml}$ & All beats & $\begin{array}{l}\text { Fr, } \\
\text { Fw }\end{array}$ & Kamrul 1195 (JUH) \\
\hline M. sylvatica. Roxb.* & Jangli aam & Tree, $\mathrm{m}$ & wd & Ad & $\mathrm{Fr}$ & Kamrul $1444(\mathrm{JUH})$ \\
\hline Holigarna caustica (Dennst.) Oken.* & Jaowa & Tree, $\mathrm{m}$ & hs & Ad & $\mathrm{T}$ & Kamrul 220 (JUH) \\
\hline Rhus succedanea $\mathrm{L}$ & Kakuasingh & Tree, 1 & wd & Ad & $\mathrm{Fr}$ & Kamrul 1377 (JUH) \\
\hline $\begin{array}{l}\text { Pegia nitida } \text { Colebr. } \\
\text { MELIACEAE Juss. }\end{array}$ & Tapir & Shrub & hs, wd & Ad & $\mathrm{Fr}, \mathrm{M}$ & Kamrul 168 (JUH) \\
\hline $\begin{array}{l}\text { Aphanamixis polystachya (Wall.) } \\
\text { R.Parker }\end{array}$ & Pitraj & Tree, 1 & wd & All beats & $\mathrm{T}, \mathrm{M}$ & Kamrul 850 (JUH) \\
\hline Azadirachta indica A.Juss. & Neem & Tree, 1 & $\mathrm{ml}$, rs & $\mathrm{Ad}$ & $\mathrm{M}, \mathrm{T}$ & Kamrul $1240(\mathrm{JUH})$ \\
\hline Swietenia mahagoni (L.) Jacq. & Mahogini & Tree, 1 & $\mathrm{ml}$, rs & $\mathrm{Ad}, \mathrm{Ka}$ & $\mathrm{T}$ & Kamrul $1283(\mathrm{JUH})$ \\
\hline S. macrophylla King & Bara mahogoni & Tree, 1 & $\mathrm{ml}$ & $\mathrm{Ad}, \mathrm{Ka}$ & $\mathrm{T}$ & Kamrul $1089(\mathrm{JUH})$ \\
\hline $\begin{array}{l}\text { Dysoxylum gotadhora (Buch.-Ham.) } \\
\text { Mabb. }\end{array}$ & Rata & Tree, 1 & ht & $\mathrm{Ad}, \mathrm{Ku}$ & $\mathrm{T}$ & Kamrul 1415 (JUH) \\
\hline
\end{tabular}


Table 1 Contd.

\begin{tabular}{|c|c|c|c|c|c|c|}
\hline Scientific name & Bangla name & Habit & Habitat & Distrib. & Uses & RSE \\
\hline Toona ciliata M. Roem. & Toon & Tree, $\mathrm{m}$ & wd & $\mathrm{Ad}$ & $\mathrm{T}, \mathrm{M}$ & Kamrul $1078(\mathrm{JUH})$ \\
\hline Walsura robusta Roxb. & Bonlichu & Tree, $\mathrm{m}$ & $\mathrm{rb}$ & Ad & $\mathrm{T}$ & Kamrul $1358(\mathrm{JUH})$ \\
\hline \multicolumn{7}{|l|}{ RUTACEAE Juss. } \\
\hline Aegle marmelos (L.) Corrêa & Bel & Tree, $\mathrm{m}$ & $\mathrm{ml}$ & Ad & $\mathrm{M}, \mathrm{Fr}$ & Kamrul 2078 (JUH) \\
\hline Acronychia pedunculata (L.) Miq. & Bon jamir & Shrub & hs & All beats & $\begin{array}{l}\mathrm{Fr} \\
\mathrm{Fw}\end{array}$ & Kamrul 541 (JUH) \\
\hline $\begin{array}{l}\text { Clausena anisata (Willd.) Hook. f. ex } \\
\text { Benth. }\end{array}$ & Kalo-maricha & Shrub & wd & All beats & - & Kamrul $112(\mathrm{JUH})$ \\
\hline Citrus medica $\mathrm{L}$. & Pani lebu & Shrub & wd & $\mathrm{Ad}, \mathrm{Ku}$ & $\mathrm{Pe}, \mathrm{M}$ & Kamrul $1171(\mathrm{JUH})$ \\
\hline Glycosmis pentaphylla (Retz.) DC. & Datmajani & Shrub & wd & $\mathrm{Ad}, \mathrm{Ku}$ & M & Kamrul 815 (JUH) \\
\hline Micromelum minutum Wight \& Arn. & Dulia & Tree, $\mathrm{s}$ & wd & All beats & M & Kamrul 244 (JUH) \\
\hline Murraya koenigii (L.) Spreng. & Curry pata & Shrub & wd & $\mathrm{Ku}$ & $\begin{array}{l}\mathrm{Fr}, \mathrm{Sp} \\
\mathrm{M}\end{array}$ & , Kamrul 1576 (JUH) \\
\hline \multicolumn{7}{|l|}{ OXALIDACEAE R. Br. } \\
\hline Averrhoa bilimbi L. & Bilimbi & Tree, $\mathrm{s}$ & $\mathrm{ml}(\mathrm{pl})$ & Ad & $\mathrm{V}$ & Kamrul $2184(\mathrm{JUH})$ \\
\hline $\begin{array}{l}\text { A. carambola } \mathrm{L} \text {. } \\
\text { ARALIACEAE Juss. }\end{array}$ & \multicolumn{5}{|c|}{ ARALIACEAE Juss. } & Kamrul $2186(\mathrm{JUH})$ \\
\hline Brassaiopsis glomerulata (Blume) Regel & Kurila & Tree, $\mathrm{s}$ & wd, hs & All beats & $\mathrm{M}, \mathrm{O}$ & Kamrul $1384(\mathrm{JUH})$ \\
\hline $\begin{array}{l}\text { Trevesia palmata (Roxb. ex Lind.) Vis. } \\
\text { APIACEAE Lindl. }\end{array}$ & Argoja & Tree, $\mathrm{m}$ & hs & Ad & $\mathrm{M}, \mathrm{O}$ & Kamrul 1095 (JUH) \\
\hline Centella asiatica $(\mathrm{L}$.$) Urb.$ & Thankuni & Herb, cr & $\mathrm{ml}$ & All beats & M & Kamrul 972 (JUH) \\
\hline Eryngium foetidum $\mathrm{L}$. & Bilati dhania & Shrub & $\mathrm{ml}(\mathrm{cl})$ & All beats & $\mathrm{Sp}, \mathrm{M}$ & Kamrul $1134(\mathrm{JUH})$ \\
\hline $\begin{array}{l}\text { Hydrocotyle sibthorpioides Lam. } \\
\text { BUDDLEJACEAE Wilh. }\end{array}$ & Gimashak & Herb, cr & wV & Ad & - & Kamrul $1581(\mathrm{JUH})$ \\
\hline $\begin{array}{l}\text { Buddleja asiatica Lour. } \\
\text { GENTIANACEAE Juss. }\end{array}$ & Neemda & Shrub & wd & All beats & $\mathrm{M}, \mathrm{Pe}$ & Kamrul 775 (JUH) \\
\hline $\begin{array}{l}\text { Canscora andrographioides Griff. ex } \\
\text { C.B.Clarke * }\end{array}$ & Andakuni & Herb, cr & $\mathrm{fv}, \mathrm{ml}$ & Ad & - & Kamrul 164 (JUH) \\
\hline $\begin{array}{l}\text { C. alata (Roth ex Roem. \& Schult.) } \\
\text { Wall. }\end{array}$ & Dhankuni & Herb, e & $\mathrm{ml}, \mathrm{rs}$ & All beats & M & Kamrul 2068 (JUH) \\
\hline $\begin{array}{l}\text { C. diffusa (Vahl) R.Br. ex Roem. \& } \\
\text { Schult. } \\
\text { APOCYNACEAE Juss. }\end{array}$ & Fusakoni & Herb, cr & $\mathrm{ml}$ & $\mathrm{Ad}, \mathrm{Ku}$ & - & Kamrul $1116(\mathrm{JUH})$ \\
\hline Alstonia scholaris (L.) R. Br. & Chhatim & Tree, 1 & wd & $\mathrm{Ka}$ & $\begin{array}{l}\mathrm{M} \\
\mathrm{Sw}\end{array}$ & Kamrul $2138(\mathrm{JUH})$ \\
\hline Holarrhena pubescens Wall. ex G. Don & Kurchi & Shrub & sj & Ad & M & Kamrul 1823 (JUH) \\
\hline Ichnocarpus frutescens (L.) W.T. Aiton & Parallia lata & Herb, c & fe & All beats & M & Kamrul 431 (JUH) \\
\hline Rauvolfia serpentina (L.) Benth. ex Kurz * & Sarpagandha & Herb, e & ht & Ad & M & Kamrul 737 (JUH) \\
\hline $\begin{array}{l}\text { Tabernaemontana divaricata (L.) R.Br. } \\
\text { ex Roem. \& Schult. }\end{array}$ & Tagar & Shrub & sj & All beats & M & Kamrul 21 (JUH) \\
\hline ASCLEPIADACEAE Borkh. & & & & & & \\
\hline Gymnema acuminatum Wall. & Nimakumina & Liana & $\mathrm{fe}$ & Ad & & Kamrul 981 (JUH) \\
\hline $\begin{array}{l}\text { Hoya parasitica Wall. ex Wight } \\
\text { SOLANACEAE Juss. }\end{array}$ & Pargacha & Herb, ps & wd & All beats & M & Kamrul $1276(\mathrm{JUH})$ \\
\hline Datura metel L. & Dhutra & Shrub & $\mathrm{sj}$ & Ad & M & Kamrul 2079 (JUH) \\
\hline Nicotiana plumbaginifolia Viv. & Ban tamak & Herb, e & & All beats & - & Kamrul 2198 (JUH) \\
\hline Solanum torvum $\mathrm{Sw}$. & Gota begun & Herb, e & $\mathrm{sj}$ & All beats & - & Kamrul $2196(\mathrm{JUH})$ \\
\hline
\end{tabular}


Table 1 Contd.

\begin{tabular}{|c|c|c|c|c|c|c|}
\hline Scientific name & Bangla name & Habit & Habitat & Distrib. & Uses & RSE \\
\hline S. americanum Mill. & Tit-begun & Herb, e & sj & All beats & M & Kamrul 1348 (JUH) \\
\hline Physalis angulata $\mathrm{L}$. & Futka & Herb, e & rs & Ad & - & Kamrul 2200 (JUH) \\
\hline CONVOLVULACEAE Juss. & & & & & & \\
\hline Argyreia argentea (Roxb.) Sweet & Boro rupatala & Herb, c & sj & All beats & - & Kamrul 394 (JUH) \\
\hline A. capitiformis (Poir.) Ooststr. & Bijtarak & Herb, c & fe & All beats & M & Kamrul 124 (JUH) \\
\hline Cuscuta reflexa Roxb. & Sarnalata & Herb, ps & $\mathrm{ml}$ & All beats & M & Kamrul 1463 (JUH) \\
\hline Evolvulus nummularius (L.) L. & Bhuiokua & Herb, $\mathrm{Cr}$ & sj & Ad & M & Kamrul 2146 (JUH) \\
\hline Ipomoea aquatica Forssk. & Kolmishak. & Herb, a & $\mathrm{ml}$ & Ad & $\mathrm{V}$ & Kamrul 2131 (JUH) \\
\hline I. alba $\mathrm{L}$. & Dudh kolmi & Herb, cr & $\mathrm{rb}$ & $\mathrm{Ad}$ & M & Kamrul 2130 (JUH) \\
\hline I. batatas (L.) Lam. & Shakalu & Herb, cr & $\mathrm{rb}$ & Ad & M & Kamrul 2132 (JUH) \\
\hline I. cairica $($ L.) Sweet & Rail lata & Herb, cr & sj & $\mathrm{Ad}$ & - & Kamrul 1049 (JUH) \\
\hline I. carnea Jacq. & Dhol kolmi & Shrub & $\mathrm{ml}$ & Ad & - & Kamrul 980 (JUH) \\
\hline $\begin{array}{l}\text { Merremia umbellata (L.) Hallier f. } \\
\text { MENYANTHACEAE Dumort. }\end{array}$ & Sada kalmi & Herb, cr & rs, fv & All beats & M & Kamrul 17 (JUH) \\
\hline $\begin{array}{l}\text { Nymphoides hydrophylla (Lour.) Kuntze } \\
\text { HYDROPHYLLACEAE R. Br. }\end{array}$ & Chadmala & Herb, a & $\mathrm{ml}$ & $\mathrm{Ad}, \mathrm{Ku}$ & $\mathrm{Fd}$ & Kamrul $728(J U H)$ \\
\hline $\begin{array}{l}\text { Hydrolea zeylanica (L.) Vahl } \\
\text { BORAGINACEAE Juss. }\end{array}$ & Kasschara & Herb, a & $\mathrm{ml}$ & All beats & M & Kamrul 901 (JUH) \\
\hline Cordia dichotoma G. Forst. & Boula & Tree, $\mathrm{m}$ & $\mathrm{rb}$ & All beats & M & Kamrul 2144 (JUH) \\
\hline $\begin{array}{l}\text { Heliotropium indicum } \text { L. } \\
\text { VERBENACEAE J.St.-Hil. }\end{array}$ & Hatisur & Herb, e & $\mathrm{sj}$ & All beats & M & Kamrul 2147 (JUH) \\
\hline $\begin{array}{l}\text { Lantana camara } \mathrm{L} \text {. } \\
\text { LAMIACEAE Martinov }\end{array}$ & Kutuskanta & Shrub & wd, fe, ml & All beats & M & Kamrul 16 (JUH) \\
\hline Callicarpa arborea Roxb. & Barmala & Tree & ht & All beats & M & Kamrul 499 (JUH) \\
\hline C. longifolia Lam. & Lamarck & Shrub & wd & $\mathrm{Ad}, \mathrm{Ku}$ & - & Kamrul 1354 (JUH) \\
\hline Clerodendrum indicum (L.) Kuntze & Bamunhatti & Shrub & wd, fe & Ad & M & Kamrul 1059 (JUH) \\
\hline C. infortunatum $\mathrm{L}$. & Bhat & Shrub & wd, fe & All beats & - & Kamrul 13 (JUH) \\
\hline C. laevifolium Blume & Mali bong & Shrub & wd, fe & $\mathrm{Ad}$ & $\mathrm{O}$ & Kamrul 59 (JUH) \\
\hline Gmelina arborea Roxb. ex Sm. & Gamari & Tree, $\mathrm{m}$ & hs & All beats & $\mathrm{T}, \mathrm{M}$ & Kamrul 1219 (JUH) \\
\hline $\begin{array}{l}\text { Gomphostema salarkhaniana Khanam \& } \\
\text { Hassan * }\end{array}$ & Kanimala & Herb, e & wd & Ad & - & Kamrul 84 (JUH) \\
\hline Hyptis brevipes Poit. & Gol-tokma & Herb, e & sj, wd & All beats & M & Kamrul 43 (JUH) \\
\hline H. suaveolens (L.) Poit. & Tokma & Herb, e & $\mathrm{sj}$ & $\mathrm{Ad}, \mathrm{Ka}$ & M & Kamrul 994 (JUH) \\
\hline Leucas zeylanica (L.) W. T. Aiton & Dondokalosh & Herb, e & $\mathrm{ml}$ & All beats & M & Kamrul 256 (JUH \\
\hline $\begin{array}{l}\text { Mosla dianthera (Buch.-Ham. ex Roxb.) } \\
\text { Maxim. }\end{array}$ & Moshla & Herb, e & sj & $\mathrm{Ka}$ & M & Kamrul 1064 (JUH) \\
\hline Ocimum gratissimum $\mathrm{L}$ & Ram tulsi & Shrub & $\mathrm{ml}$ & All beats & M & Kamrul 1131 (JUH) \\
\hline O. tenuiflorum $\mathrm{L}$. & Kalo tulsi & Herb, e & sj & Ad & M & Kamrul 2061 (JUH) \\
\hline Pogostemon auricularius (L.) Hassk. & Aripachuli & Herb, e & wt & $\mathrm{Ad}, \mathrm{Ku}$ & M & Kamrul 37 (JUH) \\
\hline $\begin{array}{l}\text { Rotheca serrata }(\mathrm{L} .) \text { Steane \& Mabb. } \\
\text { OLEACEAE Hoffeanns. \& Link }\end{array}$ & Bamanhati & Shrub & wd & Ad & M & Kamrul 120 (JUH) \\
\hline Jasminum sambac (L.) Aiton & Beli & Shrub & fe & All beats & $\mathrm{O}$ & Kamrul 1004 (JUH) \\
\hline J. scandens (Retz.) Vahl & Jua & Shrub & hs & All beats & - & Kamrul 142 (JUH) \\
\hline Myxopyrum smilacifolium (Wall.) Blume & Chiknabizi & Herb, e & wd & All beats & - & Kamrul 607 (JUH) \\
\hline Nyctanthes arbor-tristis L. & Sheuly & Tree, $\mathrm{m}$ & wd & Ad & $\mathrm{T}, \mathrm{M}$ & Kamrul 1149 (JUH) \\
\hline Premna esculenta Roxb. & Lalong & Shrub & ht, rs & All beats & $\mathrm{V}, \mathrm{O}$ & Kamrul 323 (JUH) \\
\hline Tectona grandis L.f. & Shegun & Tree, 1 & wd & All beats & $\mathrm{T}$ & Kamrul 489 (JUH) \\
\hline Vitex negundo L. & Nishinda & Tree, $\mathrm{s}$ & $\mathrm{rb}$ & All beats & M & Kamrul 588 (JUH) \\
\hline
\end{tabular}


Table 1 Contd.

\begin{tabular}{|c|c|c|c|c|c|c|}
\hline Scientific name & Bangla name & Habit & Habitat & Distrib. & Uses & RSE \\
\hline V. peduncularis Wall. ex Schauer & Awal & Tree, $\mathrm{m}$ & ht, hs & All beats & $\mathrm{T}$ & Kamrul 1257 (JUH) \\
\hline$V$. pinnata $\mathrm{L}$. & Seliawal & Tree, $\mathrm{m}$ & hs, fe & Ad & - & Kamrul $1413(\mathrm{JUH})$ \\
\hline \multicolumn{7}{|l|}{ PLANTAGINACEAE Juss. } \\
\hline Limnophila rugosa (Roth) Merr. & Bandha keshori & Herb, e & $\mathrm{ml}$ & All beats & $\mathrm{M}$ & Kamrul $1128(\mathrm{JUH})$ \\
\hline Mecardonia procumbens (Mill.) Small & Ada birni & Herb, e & $\mathrm{ml}$ & Ad & - & Kamrul 135 (JUH) \\
\hline Scoparia dulcis L. & Bondhone & Herb, e & $\mathrm{ml}, \mathrm{rs}$ & All beats & M & Kamrul 796 (JUH) \\
\hline \multicolumn{7}{|c|}{ LINDERNIACEAE Borsch, Kai Müll. \& Eb. Fisch. } \\
\hline Lindernia ciliata (Colsm.) Pennell & Bhui papri & Herb, pr & $\mathrm{rb}, \mathrm{fe}, \mathrm{hs}$ & All beats & - & Kamrul 755 (JUH) \\
\hline L. antipoda (L.) Alston & & Herb, pr & sj, gl & All beats & - & Kamrul 373 (JUH) \\
\hline $\begin{array}{l}\text { Torenia fournieri Linden ex E.Fourn. } \\
\text { OROBANCHACEAE Vent. }\end{array}$ & Neritoren & Herb, pr & sj & All beats & $\mathrm{O}$ & Kamrul 19 (JUH) \\
\hline $\begin{array}{l}\text { Aeginetia indica } \mathrm{L} . \\
\text { ACANTHACEAE Juss. }\end{array}$ & Agienata & Herb, ps & hs & Ad & $\mathrm{O}$ & Kamrul $1380(\mathrm{JUH})$ \\
\hline Acanthus leucostachyus Wall. ex Nees * & Kastacha & Shrub & wd & Ad & M & Kamrul 1217 (JUH) \\
\hline $\begin{array}{l}\text { Andrographis paniculata (Burm.f.) Wall. } \\
\text { ex Nees * }\end{array}$ & Kalomegh & Herb, e & $\mathrm{sj}$ & Ad, Ka & M & Kamrul 2081 (JUH) \\
\hline $\begin{array}{l}\text { Blepharis integrifolia (L.f.) E.Mey. \& } \\
\text { Drège ex Schinz. }\end{array}$ & Deshi blephar & Herb, e & sj & Ad & - & Kamrul 1143 (JUH) \\
\hline Eranthemum strictum Colebr. ex Roxb. & Khara murali & Shrub & wd & All beats & - & Kamrul 1161 (JUH) \\
\hline $\begin{array}{l}\text { Hygrophila polysperma (Roxb.) T. } \\
\text { Anderson }\end{array}$ & Murmura & Herb, e & $\mathrm{ml}$ & Ad & - & Kamrul 1661 (JUH) \\
\hline Justicia adhatoda $\mathrm{L}$. & Bashak & Shrub & $\mathrm{fe}$ & Ad & M & Kamrul 2136 (JUH) \\
\hline J. diffusa Willd. & Pitapapra & Herb, e & rs, sj & Ad & & Kamrul 2135 (JUH) \\
\hline $\begin{array}{l}\text { Lepidagathis incurva Buch.-Ham. ex } \\
\text { D.Don }\end{array}$ & Linagathis & Herb, e & sj & All beats & - & Kamrul 905 (JUH) \\
\hline Nelsonia canescens (Lam.) Spreng. & Paramul & Herb, e & $\mathrm{ml}$ & All beats & - & Kamrul 182 (JUH) \\
\hline Phlogacanthus curviflorus (Wall.) Nees & Agnilora & Shrub & fe & Ad & $\mathrm{O}$ & Kamrul 1163 (JUH) \\
\hline P. thyrsiformis (Roxb. ex Hardw.) Mabb. & Rambasak & Shrub & $\mathrm{fe}$ & Ad & M & Kamrul 108 (JUH) \\
\hline P. tubiflorus Nees & Agnibasak & Shrub & fe & Ad & - & Kamrul 121 (JUH) \\
\hline $\begin{array}{l}\text { Phaulopsis imbricata (Forssk.) Sweet } \\
\text { Hort. }\end{array}$ & Kantasi & Herb, e & $\mathrm{sj}$ & All beats & - & Kamrul 2067 (JUH) \\
\hline Rungia pectinata $(\mathrm{L}$.$) Nees$ & Pindi & Herb, pr & $\mathrm{ml}$ & All beats & M & Kamrul 1975 (JUH) \\
\hline Staurogyne argentea Wall. & - & Herb, e & hf & All beats & - & Kamrul 811 (JUH) \\
\hline S. polybotrya Kuntze & Polygyne & Herb, e & $\mathrm{ml}$ & Ad & - & Kamrul 1124 (JUH) \\
\hline S. zeylanica Kuntze & Cylongyne & Herb, e & ht & Ad & - & Kamrul 1579 (JUH) \\
\hline Strobilanthes scaber Nees & Khaskhasabila & Herb, e & $\mathrm{sj}$ & All beats & - & Kamrul 132 (JUH) \\
\hline $\begin{array}{l}\text { Thunbergia grandiflora (Roxb. ex Rottl.) } \\
\text { Roxb. }\end{array}$ & Neel lata & Herb, cl & $\mathrm{sp}$ & All beats & $\mathrm{O}$ & Kamrul 638 (JUH) \\
\hline BIGNONIACEAE Juss. & & & & & & \\
\hline $\begin{array}{l}\text { Stereospermum tetragonum DC. } \\
\text { CAMPALUNACEAE Juss. }\end{array}$ & Awal & Tree, 1 & wd & Ad & - & Kamrul 1804 (JUH) \\
\hline $\begin{array}{l}\text { Lobelia zeylanica } \mathrm{L} \text {. } \\
\text { RUBIACEAE Juss. }\end{array}$ & Cylon lobel & Herb, pr & sj & All beats & - & Kamrul $883(\mathrm{JUH})$ \\
\hline Catunaregam spinosa (Thunb.) Tirveng. & Mankanta & Shrub & $\mathrm{rb}$ & Ad & M & Kamrul 1322 (JUH) \\
\hline $\begin{array}{l}\text { Dentella repens (L.) J.R. Forst. \& } \\
\text { G.Forst. }\end{array}$ & Bhuipat & Herb, pr & sj & $\mathrm{Ad}, \mathrm{Ku}$ & - & Kamrul 45 (JUH) \\
\hline Gardenia coronaria Buch.-Ham. & Sitgach & Tree, $\mathrm{m}$ & hs & Ad & $\mathrm{T}, \mathrm{Fr}$ & Kamrul 1254 (JUH) \\
\hline $\begin{array}{l}\text { Scleromitrion scabrum (Wall. ex Kurz) } \\
\text { Neupane \& N. Wikstrom }\end{array}$ & - & Herb, e & hs & $\mathrm{Ad}, \mathrm{Ku}$ & - & Kamrul 46 (JUH) \\
\hline
\end{tabular}


Table 1 Contd.

\begin{tabular}{|c|c|c|c|c|c|c|}
\hline Scientific name & Bangla name & Habit & Habitat & Distrib. & Uses & RSE \\
\hline Hedyotis scandens Roxb. & Bish lata & Herb, pr & wd & All beats & $\mathrm{M}$ & Kamrul 1486 (JUH) \\
\hline H. verticillata (L.) Lamk. & - & Herb, pr & wd & All beats & - & Kamrul 1559 (JUH) \\
\hline Ixora acuminata Roxb. & Nata rangan & Shrub & wd & All beats & $\mathrm{O}$ & Kamrul $1164(\mathrm{JUH})$ \\
\hline I. coccinea $\mathrm{L}$. & Rangan & Shrub & wd & $\mathrm{Ad}, \mathrm{Ku}$ & $\mathrm{O}$ & Kamrul $1288(\mathrm{JUH})$ \\
\hline I. pavetta Andr. & Ganghalrangan & Shrub & wd & All beats & - & Kamrul 434 (JUH) \\
\hline I. spectabilis Wall. ex G.Don & Shum rangan & Shrub & wd & $\mathrm{Ad}, \mathrm{Ku}$ & - & Kamrul 31 (JUH) \\
\hline Knoxia sumatrensis (Retz.) DC & Sumatranoxi & Herb, cr & wd & Ad & - & $\begin{array}{l}\text { S.N.Uddin N4744 } \\
\text { (DACB) }\end{array}$ \\
\hline Lasianthus chrysoneurus (Korth.) Miq. & Sony lasi & Shrub & wd & Ad & - & Kamrul 1167 (JUH) \\
\hline Morinda angustifolia Roxb. & Jangli basok & Tree, $\mathrm{s}$ & sj & All beats & M & Kamrul 171 (JUH) \\
\hline Mussaenda roxburghii Hook. f. & Sil daura & Shrub & hs & All beats & M & Kamrul 1505 (JUH) \\
\hline M. frondosa $\mathrm{L}$. & Nagabali & Shrub & wd & All beats & M & Kamrul 1259 (JUH) \\
\hline M. macrophylla Wall. & $\begin{array}{l}\text { Baropata } \\
\text { muchenda }\end{array}$ & Shrub & wd & All beats & - & Kamrul 25 (JUH) \\
\hline Mussaenda sp. & - & Shrub, cl & wd & Ad & - & Kamrul $630(\mathrm{JUH})$ \\
\hline Mycetia longifolia (Wall.) Kuntze & Mycetelon & Shrub & sj & $\mathrm{Ad}, \mathrm{Ku}$ & - & Kamrul 401 (JUH) \\
\hline Myrioneuron nutans R. Br. ex Kurz & Natanuran & Shrub & wd & Ad & - & Kamrul $1518(\mathrm{JUH})$ \\
\hline Mitragyna parviflora (Roxb.) Korth. & Dakrom & Tree, $\mathrm{m}$ & ht & Ad & - & Kamrul 2243 (JUH) \\
\hline Neolamarckia cadamba (Roxb.) Bosser & Kadam & Tree, 1 & rs & $\mathrm{Ad}, \mathrm{Ku}$ & $\mathrm{O}$ & Kamrul 297 (JUH) \\
\hline Ophiorrhiza mungos L. & Gandahanakuli. & Herb, e & sj & All beats & M & Kamrul 23 (JUH) \\
\hline Pavetta indica $\mathrm{L}$. & Kathchapa & Shrub & wd & Ad & M & Kamrul 1309 (JUH) \\
\hline P. polyantha (Hook.f.) R. Br. ex Bremek. & Polinakli & Shrub & wd & Ad & - & Kamrul 1609 (JUH) \\
\hline Psychotria adenophylla Wall. & Baro sudma & Shrub & wd & All beats & M & Kamrul 131 (JUH) \\
\hline P. calocarpa Kurz & Ranga bhutta & Shrub & wd & Ad & M & Kamrul 917 (JUH) \\
\hline Coffea benghalensis B.Heyne ex Schult. & Bonnya kofee & Shrub & hs & Ad & - & Kamrul 1617 (JUH) \\
\hline Richardia scabra L. & Nakli ipecac & Herb, e & sj & Ad & - & Kamrul 880 (JUH) \\
\hline $\begin{array}{l}\text { Oxyceros kunstleri (King \& Gamble) } \\
\text { Tirveng. }\end{array}$ & Ichuri & Shrub & wd & Ad & M & Kamrul 874 (JUH) \\
\hline $\begin{array}{l}\text { Wendlandia grandis (Hook. f.) Cowan } \\
\text { ASTERACEAE Bercht. \& J. Presl }\end{array}$ & Tulaload & Tree, 1 & wd & Ad & - & Kamrul 149 (JUH) \\
\hline Acmella caulirhiza Delile & Mahatitinga & Herb, pr & fe & All beats & - & Kamrul 194 (JUH) \\
\hline Adenostemma lavenia (L.) Kuntze & Baro-kesuti & Herb, e & $\mathrm{fe}$ & All beats & - & Kamrul 963 (JUH) \\
\hline Ageratum conyzoides $\mathrm{L}$. & Fulkuri & Herb, e & $\mathrm{rs}, \mathrm{sj}, \mathrm{ml}$ & All beats & M & Kamrul 08 (JUH) \\
\hline Blumea lacera (Burm.f.) DC. & Barokukshim & Herb, pr & $\mathrm{rs}, \mathrm{sj}$ & All beats & M & Kamrul $1186(\mathrm{JUH})$ \\
\hline $\begin{array}{l}\text { Crassocephalum crepidioides (Benth.) } \\
\text { S. Moore }\end{array}$ & Duubbecrepi & Herb, e & fe & All beats & $\mathrm{M}, \mathrm{V}$ & Kamrul 417 (JUH) \\
\hline $\begin{array}{l}\text { Chromolaena odorata (L.) R.M.King \& } \\
\text { H. Rob. }\end{array}$ & Rail lata & Shrub & $\mathrm{sj}, \mathrm{fe}$ & All beats & M & Kamrul 923 (JUH) \\
\hline Cosmos sulphureus Cav. & Tara gada & Herb, e & $\mathrm{ml}(\mathrm{pl})$ & $\mathrm{Ka}$ & $\mathrm{O}$ & Kamrul $2251(\mathrm{JUH})$ \\
\hline Cyanthillium cinereum (L.) H. Rob. & Shialmutra & Herb, e & $\mathrm{ml}$ & All beats & M & Kamrul 2142 (JUH) \\
\hline Eclipta prostrata $(\mathrm{L}.) \mathrm{L}$. & Kalokeshi & Herb, pr & $\mathrm{ml}, \mathrm{fe}$ & All beats & M & Kamrul 357 (JUH) \\
\hline Elephantopus scaber $\mathrm{L}$. & Hastipadi & Herb, e & $\mathrm{ml}, \mathrm{fe}, \mathrm{sj}$ & All beats & M & Kamrul 567 (JUH) \\
\hline Enhydra fluctuans Lour. & Helencha & Herb, aq & $\mathrm{ml}$ & All beats & & Kamrul 2145 (JUH) \\
\hline Grangea maderaspatana (L.) Poir. & Namuti & Herb, e & $\mathrm{rb}$ & All beats & M & Kamrul 1192 (JUH) \\
\hline Launaea asplenifolia (Willd.) Hook.f. & Tikadana & Herb, e & hs & Ad & & Kamrul 1604 (JUH) \\
\hline Mikania micrantha Kunth & Assam lata & Herb, c & $\mathrm{ml}$ & All beats & M & Kamrul 74 (JUH) \\
\hline $\begin{array}{l}\text { Pseudognaphalium luteoalbum (L.) } \\
\text { Hilliard \& B.L. Burtt }\end{array}$ & Bara kamra & Herb, e & sj & Ad & M & Kamrul 155 (JUH) \\
\hline Synedrella nodiflora (L.) Gaertn. & Nakphul & Herb, e & rs, fe & All beats & - & Kamrul $2141(\mathrm{JUH})$ \\
\hline Xanthium strumarium L. & Ghagra & Herb, e & $\mathrm{fe}$ & Ad & - & Kamrul 1753 (JUH) \\
\hline
\end{tabular}


Table 1 Contd.

\begin{tabular}{|c|c|c|c|c|c|c|}
\hline Scientific name & Bangla name & Habit & Habitat & Distrib. & Uses & RSE \\
\hline \multicolumn{7}{|l|}{ LILIOPSIDA Batsch } \\
\hline \multicolumn{7}{|l|}{ HYDROCHARITACEAE Juss. } \\
\hline $\begin{array}{l}\text { Blyxa japonica (Miquel) Maxim. ex } \\
\text { Asch. \& Gürke }\end{array}$ & Japani blixa & Herb, aq & $\mathrm{ml}$ & $\mathrm{Ka}$ & - & Kamrul 1503 (JUH) \\
\hline Ottelia alismoides (L.) Pers. & Panikola & Herb, aq & $\mathrm{ml}$ & $\mathrm{Ku}$ & Fr, M & Kamrul 1504 (JUH) \\
\hline \multicolumn{7}{|l|}{ ARECACEAE Bercht. \& J.Presl } \\
\hline Areca catechu $\mathrm{L}$. & Supari & Tree, $\mathrm{m}$ & $\mathrm{ml}(\mathrm{pl})$ & Ad & $\mathrm{M}, \mathrm{T}$ & Kamrul 2108 (JUH) \\
\hline Borassus flabellifer $\mathrm{L}$. & Tal & Tree, 1 & $\mathrm{ml}(\mathrm{pl})$ & Ad & $\begin{array}{l}\mathrm{Fr}, \\
\mathrm{Du}, \mathrm{Ju}\end{array}$ & Kamrul $2110(\mathrm{JUH})$ \\
\hline Caryota urens $\mathrm{L}$. & Chau-gota & Tree, $\mathrm{m}$ & ht & Ad & - & Kamrul 625 (JUH) \\
\hline Calamus tenuis Roxb. & Jali bet & Herb, cl & wd & All beats & $\mathrm{Du}$ & Kamrul $1558(\mathrm{JUH})$ \\
\hline C. erectus Roxb. * & Sungota & Herb, e & wd & $\mathrm{Ad}, \mathrm{Ku}$ & $\mathrm{Du}$ & Kamrul 574 (JUH) \\
\hline C. longisetus Griff. * & Bet & Shrub & wd & $\mathrm{Ka}$ & $\mathrm{Du}$ & $\begin{array}{l}\text { S.N.Uddin N4485 } \\
\text { (DACB) }\end{array}$ \\
\hline Cocos nucifera $\mathrm{L}$. & Narikel & Tree, 1 & $\mathrm{ml}$ & Ad & $\begin{array}{l}\mathrm{Fr}, \mathrm{Ol}, \\
\mathrm{Du}\end{array}$ & Kamrul 2111 (JUH) \\
\hline Daemonorops jenkinsiana (Griff.) Mart. & Golla & Shrub & hs & Ad & $\mathrm{Du}$ & Kamrul 1809 (JUH) \\
\hline Phoenix sylvestris (L.) Roxb. & Deshi khejur & Tree, $\mathrm{m}$ & wd & Ad & $\mathrm{Du}, \mathrm{Ju}$ & Kamrul $2212(\mathrm{JUH})$ \\
\hline \multicolumn{7}{|l|}{ PANDANACEAE R.Br. } \\
\hline Pandanus foetidus Roxb. & Keya kanta & Shrub & fv & Ad & - & Kamrul $1042(\mathrm{JUH})$ \\
\hline \multicolumn{7}{|l|}{ ARACEAE Juss. } \\
\hline Aglaonema hookerianum Schott * & Nimahook & Herb, e & hs, rb & Ad & $\mathrm{M}$ & Kamrul $1597(\mathrm{JUH})$ \\
\hline Alocasia cucullata (Lour.) G. Don & Bishkachu & Herb, e & hs, rb & All beats & M & Kamrul 225 (JUH) \\
\hline A. macrorrhizos (L.) G. Don & Mankachu & Herb, e & $\mathrm{rb}, \mathrm{ml}$ & Ad & M & Kamrul 2073 (JUH) \\
\hline Amorphophallus bulbifer (Roxb.) Blume & Owl & Herb, e & hs, ml & $\mathrm{Ad}, \mathrm{Ku}$ & $\mathrm{V}$ & Kamrul 250 (JUH) \\
\hline Colocasia esculenta $($ L.) Schott & Jangli kachu & Herb, e & $\mathrm{rb}, \mathrm{rf}$ & All beats & $\mathrm{V}, \mathrm{M}$ & Kamrul $1373(\mathrm{JUH})$ \\
\hline C. gigantea (Blume) Hook.f. & Salad kachu & Herb, e & $\mathrm{ml}$ & Ad & $\mathrm{V}$ & Kamrul 2031 (JUH) \\
\hline Homalomena aromatica (Spreng.) Schott & t Gandhabi kochu & Herb, e & hs & Ad & $\mathrm{V}$ & Kamrul 325 (JUH) \\
\hline Lasia spinosa $(\mathrm{L}$.$) Thwaites$ & Kanta kachu & Herb, e & wd & $\mathrm{Ad}, \mathrm{Ku}$ & $\mathrm{V}, \mathrm{M}$ & Kamrul 624 (JUH) \\
\hline Pistia stratiotes $\mathrm{L}$. & Topapana & Herb, aq & $\mathrm{ml}$ & $\mathrm{Ku}$ & M & Kamrul 2002 (JUH) \\
\hline Pothos scandens L. & Hatilata & Liana, ep & $\mathrm{sj}$ & All beats & M & Kamrul 9 (JUH) \\
\hline $\begin{array}{l}\text { Epipremnum aureum (Linden \& André) } \\
\text { G.S. Bunting }\end{array}$ & Money plant & Liana & $\mathrm{ml}$ & Ad & $\mathrm{O}$ & Kamrul 2004 (JUH) \\
\hline Rhaphidophora glauca (Wall.) Schott & Fidoka & Herb, cl & wd & Ad & - & Kamrul $1386(\mathrm{JUH})$ \\
\hline Steudnera colocasioides Hook. f. * & Biskachu & Herb, e & $\mathrm{ml}$ & Ad & $\mathrm{V}$ & Kamrul 2027 (JUH) \\
\hline Typhonium flagelliforme (Lodd.) Blume & Ghechu & Herb, e & sj, wt & Ad & & Kamrul 2205 (JUH) \\
\hline T. trilobatum (L.) Schott & Ghetkul & Herb, e & sj & Ad & $\mathrm{V}$ & Kamrul 2207 (JUH) \\
\hline \multicolumn{7}{|l|}{ LEMNACEAE Martinov } \\
\hline Lemna perpusilla Torr. & Khudipana & Herb, aq & wt & Ad & $\begin{array}{l}\mathrm{Fd}, \\
\mathrm{Gm}\end{array}$ & Kamrul $2244(\mathrm{JUH})$ \\
\hline $\begin{array}{l}\text { COMMELINACEAE Mirb. } \\
\text { Amischotolype mollissima (Blume) } \\
\text { Hassk. }\end{array}$ & Molosima & Herb, pr & hs, rb & $\mathrm{Ad}, \mathrm{Ku}$ & - & Kamrul 399 (JUH) \\
\hline Commelina diffusa Burm.f. & Monayna kanshira & Herb, e & hs, rb & All beats & M & Kamrul $764(\mathrm{JUH})$ \\
\hline C. erecta $\mathrm{L}$. & Jata kanchira & Herb, e & sj & Ad & $\mathrm{V}$ & Kamrul 283 (JUH) \\
\hline Floscopa scandens Lour. & - & Herb, $p$ & $\mathrm{fv}$ & All beats & M & Kamrul $1024(\mathrm{JUH})$ \\
\hline Murdannia nudiflora (L.) Brenan & Kureli & Herb, e & fe, rs & All beats & - & Kamrul 91 (JUH) \\
\hline Pollia secundiflora (Blume) Bakh.f. & Kandopoli & Herb, e & wd & Ad & - & Kamrul 809 (JUH) \\
\hline
\end{tabular}


Table 1 Contd.

\begin{tabular}{|c|c|c|c|c|c|c|}
\hline Scientific name & Bangla name & Habit & Habitat & Distrib. & Uses & RSE \\
\hline \multicolumn{7}{|l|}{ ERIOCAULACEAE Martinov } \\
\hline Eriocaulon quinquangulare $\mathrm{L}$. & Guri & Herb, e & $\mathrm{ml}$ & All beats & - & Kamrul $762(\mathrm{JUH})$ \\
\hline JUNCACEAE Juss. & & & & & & \\
\hline Juncus prismatocarpus $\mathrm{R} . \mathrm{Br}$. & Atoshi junca & Herb, e & $\mathrm{rb}$ & Ad & - & Kamrul 2247 (JUH) \\
\hline \multicolumn{7}{|l|}{ CYPERACEAE Juss. } \\
\hline Bulbostylis barbata (Rottb.) C.B. Clarke. & Balbobata & Herb, e & wd & All beats & - & Kamrul 1607 (JUH) \\
\hline Cyperus compactus Retz. & Bandorghasi & Herb, e & sj, gl & All beats & $\mathrm{Sb}$ & Kamrul 2109 (JUH) \\
\hline C. iria $\mathrm{L}$. & Barachucha & Herb, e & $\mathrm{gl}$ & Ad & $\mathrm{Sb}$ & Kamrul 1125 (JUH) \\
\hline C. laxus Lam. & Alga ghasi & Herb, e & ht & All beats & $\mathrm{Sb}$ & Kamrul 63 (JUH) \\
\hline C. pilosus Vahl & Pasham kathai & Herb, e & fe & All beats & $\mathrm{Sb}$ & Kamrul 193 (JUH) \\
\hline C. tenuispica Steud. & Paikamutha & Herb, e & sj, rs & All beats & $\mathrm{Sb}$ & Kamrul 452 (JUH) \\
\hline Eleocharis geniculata (L.) Roem. \& Schult. & Joraghasi & Herb, e & $\mathrm{fe}, \mathrm{gl}$ & Ad & - & Kamrul 447 (JUH) \\
\hline Fimbristylis schoenoides (Retz.) Vahl & Kesari malanga & Herb, e & gl & All beats & - & Kamrul 1456 (JUH) \\
\hline Hypolytrum nemorum (Vahl) Spreng. & Trumram ghasi & Herb, e & fe, $g l$ & All beats & - & Kamrul 1269 (JUH) \\
\hline $\begin{array}{l}\text { Kyllinga nemoralis (J.R.Forst. \& } \\
\text { G.Forst.) Dandy ex Hutch. \& Dalziel }\end{array}$ & Subasinirbisa & Herb, e & $\mathrm{sj}, \mathrm{fe}, \mathrm{gl}$ & Ad & $\mathrm{Fd}$ & Kamrul 2215 (JUH) \\
\hline K. brevifolia Rottb. & Shabujnirbisa & Herb, e & rs, gl & All beats & $\mathrm{Fd}$ & Kamrul 64 (JUH) \\
\hline K. bulbosa P.Beauv. & Golanirbisa & Herb, e & rs, gl, & Ad & $\mathrm{Fd}$ & Kamrul 2217 (JUH) \\
\hline Pycreus polystachyos (Rottb.) P. Beauv. & Paikpoli ghasi & Herb, e & $\mathrm{fv}, \mathrm{sj}$ & Ad & $\mathrm{Sb}$ & Kamrul 446 (JUH) \\
\hline Scleria levis Retz. & Rialevi ghasi & Herb, e & gl & All beats & - & Kamrul 546 (JUH) \\
\hline S. biflora Roxb. & Riaflora ghasi & Herb, e & $\mathrm{ml}, \mathrm{fe}$ & $\mathrm{Ad}, \mathrm{Ka}$ & - & Kamrul 1512 (JUH) \\
\hline S. terrestris (L.) Fassett & Dharal ghasi & Herb, e & $\mathrm{ml}, \mathrm{gl}$ & All beats & - & Kamrul 731 (JUH) \\
\hline Rhynchospora corymbosa (L.) Britton & Shonathuti ghasi & Herb, e & $\mathrm{ml}, \mathrm{gl}$ & Ad & - & Kamrul 1404 (JUH) \\
\hline \multicolumn{7}{|l|}{ POACEAE Barnhart. } \\
\hline Bambusa balcooa Roxb. & Borak bans & Herb, e & $\mathrm{ml}$ & Ad & $\mathrm{Du}$ & Kamrul 2218 (JUH) \\
\hline B. bambos (L.) Voss & Ban bans & Herb, e & $\mathrm{ml}$ & All beats & $\mathrm{Du}$ & Kamrul 2219 (JUH) \\
\hline B. polymorpha Munro & Parua & Herb, e & hs, ht & $\mathrm{Ad}, \mathrm{Ku}$ & $\mathrm{Du}$ & Kamrul 2221 (JUH) \\
\hline B. tulda Roxb. & Mirtinga & Herb, e & $\mathrm{ml}$ & All beats & $\mathrm{Du}$ & Kamrul 457 (JUH) \\
\hline Brachiaria kurzii (Hook. f.) A. Camus & Kurokti ghas & Herb, e & sj & $\mathrm{Ad}, \mathrm{Ku}$ & - & Kamrul 1550 (JUH) \\
\hline Centotheca lappacea (L.) Desv. & Centughas & Herb, e & $\mathrm{sp}$ & All beats & $\mathrm{Fd}$ & Kamrul 941 (JUH) \\
\hline Chrysopogon aciculatus (Retz.) Trin. & Premkata & Herb, e & $\mathrm{gl}, \mathrm{rs}$ & $\mathrm{Ad}, \mathrm{Ku}$ & $\mathrm{Sb}$ & Kamrul 210 (JUH) \\
\hline C. zizanioides (L.) Roberty & Khaskhas & Herb, e & rs, gl & All beats & $\mathrm{Fd}, \mathrm{Sb}$ & Kamrul 1513 (JUH) \\
\hline Cynodon dactylon (L.) Pers. & Durba ghas & Herb, cr & $\mathrm{ml}, \mathrm{rs}$, & All beats & $\begin{array}{l}\mathrm{O}, \mathrm{Fd} \\
\mathrm{Sb}\end{array}$ & Kamrul $2222(\mathrm{JUH})$ \\
\hline Cyrtococcum oxyphyllum (Steud.) Stapf & Oxycocca ghas & Herb, e & $\mathrm{ml}, \mathrm{rs}$, & $\mathrm{Ad}, \mathrm{Ka}$ & - & Kamrul 209 (JUH) \\
\hline C. patens (L.) A. Camus & Patcocca ghas & Herb, cr & $\mathrm{gl}$ & All beats & - & Kamrul 557 (JUH) \\
\hline Dendrocalamus longispathus (Kurz) Kurz & Rupai & Herb, e & $\mathrm{ml}$ & All beats & - & Kamrul 1818 (JUH) \\
\hline Dactyloctenium aegyptium (L.) Willd. & Kakpaya & Herb, e & $\mathrm{ml}, \mathrm{rs}$ & Ad & - & Kamrul 2057 (JUH) \\
\hline Digitaria ciliaris (Retz.) Koeler & Kokjachira & Herb, e & $\mathrm{fe}$ & Ad & - & Kamrul 2572 (JUH) \\
\hline Eleusine indica (L.) Gaertn. & Malankuri & Herb, e & sj & All beats & $\mathrm{Fd}$ & Kamrul 1129 (JUH) \\
\hline Eragrostis ciliaris (L.) R. Br. & Lomkoni & Herb, e & $\mathrm{ml}$ & Ad & - & Kamrul 1563 (JUH) \\
\hline E. unioloides (Retz.) Nees ex Steud. & Chirakoni & Herb, e & sj & All beats & $\begin{array}{l}\mathrm{Fd}, \\
\mathrm{Gm}\end{array}$ & Kamrul 777 (JUH) \\
\hline Imperata cylindrica (L.) Raeusch. & Chhan & Herb, e & $\mathrm{sj}$ & Ad & $\mathrm{Fd}, \mathrm{M}$ & Kamrul 211 (JUH) \\
\hline $\begin{array}{l}\text { Hildaea pallens (Sw.) C. Silva \& R.P. } \\
\text { Oliveira }\end{array}$ & - & Herb, e & $\mathrm{fv}$ & $\mathrm{Ad}$ & - & Kamrul 832 (JUH) \\
\hline Lophatherum gracile Brongn. & Lolphali ghas & Herb, e & hs, rs & All beats & $\mathrm{Fd}$ & Kamrul 635 (JUH) \\
\hline Leersia hexandra Sw. & Fulka ghas & Herb, pr & $\mathrm{ml}$ & Ad & $\mathrm{Fd}$ & Kamrul 1130 (JUH) \\
\hline Melocanna baccifera (Roxb.) Kurz & Muli & Herb, e & hs & All beats & $\mathrm{V}, \mathrm{Du}$ & Kamrul $622(\mathrm{JUH})$ \\
\hline
\end{tabular}


Table 1 Contd.

\begin{tabular}{|c|c|c|c|c|c|c|}
\hline Scientific name & Bangla name & Habit & Habitat & Distrib. & Uses & RSE \\
\hline Oryza rufipogon Griff. & Bunodhan & Herb, e & $\mathrm{ml}$ & $\mathrm{Ad}, \mathrm{Ka}$ & $\mathrm{Fd}$ & Kamrul 461 (JUH) \\
\hline Oplismenus compositus (L.) P. Beauv. & Gohur durba & Herb, e & fe, hs & All beats & - & Kamrul 2101 (JUH) \\
\hline Panicum notatum Retz. & Panita ghas & Herb, e & fe & All beats & - & Kamrul 776 (JUH) \\
\hline P. brevifolium $\mathrm{L}$. & Panibrevi ghas & Herb, e & fe & All beats & - & Kamrul 2039 (JUH) \\
\hline Paspalum conjugatum P.J.Bergius. & Moisshya ghas & Herb, e & sj, fe & $\mathrm{Ad}, \mathrm{Ka}$ & - & Kamrul 455 (JUH) \\
\hline Phragmites karka (Retz.) Trin. ex Steud. & Khakra ghas & Herb, er & $\mathrm{sj}, \mathrm{rb}$ & $\mathrm{Ad}, \mathrm{Ku}$ & Du & Kamrul 999 (JUH) \\
\hline Saccharum spontaneum L. & Kash & Herb, e & sj & Ad, Ka & - & Kamrul 2060 (JUH) \\
\hline Setaria palmifolia (J. Koenig) Stapf. & Urodhan & Herb, e & fe & $\mathrm{Ad}, \mathrm{Ku}$ & $\mathrm{Fd}, \mathrm{M}$ & Kamrul 858 (JUH) \\
\hline $\begin{array}{l}\text { Thysanolaena latifolia (Roxb. ex } \\
\text { Hornem.) Honda }\end{array}$ & Jharu phul & Herb, e & fe, sj & All beats & $\mathrm{Du}$ & Kamrul 141 (JUH) \\
\hline $\begin{array}{l}\text { Echinochloa colona (L.) Link. } \\
\text { BROMELIACEAE Juss. }\end{array}$ & Shama ghas & Herb, e & iv & Ad & $\mathrm{Fd}$ & Kamrul 1409 (JUH) \\
\hline $\begin{array}{l}\text { Ananas comosus (L.) Merr. } \\
\text { MUSACEAE Juss. }\end{array}$ & Anarash & Herb, e & $\mathrm{ml}$ & Ad & Fr & Kamrul 2087 (JUH) \\
\hline Musa paradisiaca $\mathrm{L}$. & Kola & Herb, e & $\mathrm{ml}$ & Ad, Ka & $\begin{array}{l}\mathrm{Fr}, \mathrm{V}, \\
\mathrm{M}\end{array}$ & Kamrul 2080 (JUH) \\
\hline M. acuminata Colla & Pahari kola & Herb, e & hs & Ad & Fr & Kamrul $160(\mathrm{JUH})$ \\
\hline $\begin{array}{l}\text { M. ornata } \text { Roxb. } \\
\text { ZINGIBERACEAE Martinov }\end{array}$ & Jangli kola & Herb, e & hs & $\mathrm{Ad}, \mathrm{Ku}$ & $\mathrm{Fr}, \mathrm{V}$ & Kamrul 799 (JUH) \\
\hline Amomum aromaticum Roxb. * & Alachi & Herb, e & hs, sj & All beats & $\mathrm{Fr}, \mathrm{M}$ & Kamrul 51 (JUH) \\
\hline Alpinia malaccensis (Burm. f.) Roscoe & Deotara. & Herb, e & hs & All beats & M & Kamrul 769 (JUH) \\
\hline Curcuma aromatica Salisb. & Ban haldi & Herb, e & hs & Ad & M & Kamrul $2226(\mathrm{JUH})$ \\
\hline C. caesia Roxb. & Kala holdi & Herb, e & hs & Ad & M & Kamrul 1234 (JUH) \\
\hline C. phaeocaulis Valeton & Shoti & Herb, e & hs & All beats & M & Kamrul 2075 (JUH) \\
\hline Globba bracteolata Wall. ex Baker & Dhaki globba & Herb, e & hs & Ad & M & Kamrul 22 (JUH) \\
\hline G. marantina $\mathrm{L}$. & Maran globba & Herb, e & hs & $\mathrm{Ad}, \mathrm{Ku}$ & - & Kamrul 479 (JUH) \\
\hline G. multiflora Wall. ex Baker * & Shukh globba & Herb, e & hs & All beats & - & Kamrul 247 (JUH) \\
\hline Hedychium coronarium J. Koen. & Dolon chapa & Herb, e & $\mathrm{ml}$ & Ad & $\mathrm{O}$ & Kamrul 2227 (JUH) \\
\hline H. thyrsiforme $\mathrm{Sm} . *$ & Pala ada & Herb, e & $\mathrm{ml}$ & All beats & - & Kamrul 611 (JUH) \\
\hline Zingiber officinale Roscoe & Ada & Herb, e & $\mathrm{ml}$ & $\mathrm{Ad}, \mathrm{Ka}$ & $\mathrm{Sp}, \mathrm{M}$ & Kamrul 2232 (JUH) \\
\hline $\begin{array}{l}\text { Z. zerumbet (L.) Roscoe ex Sm. } \\
\text { COSTACEAE Nakai. }\end{array}$ & Bon ada & Herb, e & $\mathrm{ml}$ & All beats & M & Kamrul $2229(\mathrm{JUH})$ \\
\hline $\begin{array}{l}\text { Hellenia speciosa (J. Koenig) Govaerts } \\
\text { CANNACEAE Juss. }\end{array}$ & Bandugi & Herb, e & sj & Ad & M & Kamrul 395 (JUH) \\
\hline $\begin{array}{l}\text { Canna indica } \mathrm{L} \text {. } \\
\text { MARANTACEAE R.Br. }\end{array}$ & Kolabati & Herb, e & $\mathrm{ml}$ & Ad & $\mathrm{M}, \mathrm{O}$ & Kamrul $2213(\mathrm{JUH})$ \\
\hline Phrynium pubinerve Blume & Pashompitali & Herb, e & fe & All beats & Du & Kamrul $176(\mathrm{JUH})$ \\
\hline P. placentarium (Lour.) Merr. & - & Herb, e & fe & $\mathrm{Ad}, \mathrm{Ku}$ & Du & Kamrul 618 (JUH) \\
\hline $\begin{array}{l}\text { Schumannianthus dichotomus (Roxb.) } \\
\text { Gagnep. }\end{array}$ & Murta & Shrub & fe & Ad & Du & Kamrul $2276(\mathrm{JUH})$ \\
\hline PONTEDERIACEAE Kunth. & & & & & & \\
\hline Eichhornia crassipes (Mart.) Solms. & Kachuripana & Herb, aq & $\mathrm{ml}$ & Ad & $\mathrm{Fd}$ & Kamrul 1904 (JUH) \\
\hline Monochoria hastata (L.) Solms & Baranukha & Herb, aq & $\mathrm{ml}$ & All beats & $\mathrm{Fd}$ & Kamrul $226(\mathrm{JUH})$ \\
\hline $\begin{array}{l}\text { M. vaginalis (Burm.f.) C. Presl } \\
\text { HAEMODORACEAE R. Br. }\end{array}$ & Bara nukha & Herb, aq & $\mathrm{ml}$ & All beats & - & Kamrul 445 (JUH) \\
\hline Peliosanthes teta Andrews & Napigach & Herb, e & wd & $\mathrm{Ad}$ & - & Kamrul $2253(\mathrm{JUH})$ \\
\hline
\end{tabular}


Table 1 Contd.

\begin{tabular}{|c|c|c|c|c|c|c|}
\hline Scientific name & Bangla name & Habit & Habitat & Distrib. & Uses & RSE \\
\hline \multicolumn{7}{|l|}{ LILIACEAE Juss. } \\
\hline Asparagus racemosus Wild. & Shatamuli & Shrub & sj & Ad & M & Kamrul 2255 (JUH) \\
\hline Crinum amoenum Ker Gawl. ex Roxb. & Gang kachu & Herb, e & hs & All beats & $\mathrm{O}, \mathrm{M}$ & Kamrul 1910 (JUH) \\
\hline $\begin{array}{l}\text { Molineria latifolia (Dryand. ex W.T. } \\
\text { Aiton) Herb. ex Kurz }\end{array}$ & Molinpasna & Herb, e & $\mathrm{sj}$ & Ad & - & Kamrul $2258(\mathrm{JUH})$ \\
\hline Gloriosa superba $\mathrm{L}$. & Ulatchandal & Herb, c & $\mathrm{ml}$ & Ad & M & Kamrul 2257 (JUH) \\
\hline $\begin{array}{l}\text { Molineria capitulata (Lour.) Herb. } \\
\text { AGAVACEAE Dumort. }\end{array}$ & Satipata & Herb, e & fe & Ad & - & Kamrul 327 (JUH) \\
\hline Dracaena spicata Roxb. & Kado drakan & Shrub & hs & $\mathrm{Ad}, \mathrm{Ku}$ & - & Kamrul 309 (JUH) \\
\hline $\begin{array}{l}\text { Sansevieria trifasciata } \text { Prain } \\
\text { TACCACEAE Dumort. }\end{array}$ & Sutahara & Herb, e & $\mathrm{ml}$ & Ad & $\mathrm{M}, \mathrm{O}$ & Kamrul 1797 (JUH) \\
\hline $\begin{array}{l}\text { Tacca integrifolia Ker-Gawl. } \\
\text { STEMONACEAE Caruel }\end{array}$ & Mati munda & Herb, e & $\mathrm{Fe}, \mathrm{sj}$ & All beats & $\mathrm{O}$ & Kamrul 158 (JUH) \\
\hline Stichoneuron membranaceum Hook. f. * & Koniron & Herb, e & $h t, f v$ & $\mathrm{Ad}, \mathrm{Ku}$ & - & Kamrul 662 (JUH) \\
\hline $\begin{array}{l}\text { Stemona tuberosa Lour. } \\
\text { SMILACACEAE Vent. }\end{array}$ & Lalguraniya alu & Herb, e & $\mathrm{fe}$ & Ad & - & Kamrul 640 (JUH) \\
\hline Smilax ovalifolia Roxb. ex D. Don & Kumari lata & Herb, cl & wd & All beats & M & Kamrul 33 (JUH) \\
\hline $\begin{array}{l}\text { S. perfoliata Lour. } \\
\text { DIOSCOREACEAE R.Br. }\end{array}$ & Kumarika & Herb, cl & hs & $\mathrm{Ad}, \mathrm{Ku}$ & - & Kamrul $1114(\mathrm{JUH})$ \\
\hline Dioscorea alata $\mathrm{L}$. & Chuprialu & Herb, cl & wd & All beats & $\mathrm{V}$ & Kamrul $2028(J U H)$ \\
\hline D. bulbifera $\mathrm{L}$. & Sora alu & Herb, cl & $\mathrm{fe}, \mathrm{sj}$ & Ad & $\mathrm{V}, \mathrm{M}$ & Kamrul 2046 (JUH) \\
\hline D. glabra Roxb. & Sora alu & Herb, cl & wd & All beats & M & Kamrul 1044 (JUH) \\
\hline D. hamiltonii Hook. f. & Thakan budo & Herb, cl & $\mathrm{sj}$ & Ad & $\mathrm{V}$ & Kamrul $2066(\mathrm{JUH})$ \\
\hline $\begin{array}{l}\text { D. pentaphylla L. } \\
\text { ORCHIDACEAE Juss. }\end{array}$ & Jum alu & Herb, cl & $\mathrm{sj}$, rs & All beats & M & Kamrul 319 (JUH) \\
\hline Aerides odorata Lour. & Churi & Herb, ep & wd & Ad & $\mathrm{O}$ & Kamrul 1327 (JUH) \\
\hline Cymbidium aloifolium (L.) Sw. * & Churi & Herb, ep & wd & $\mathrm{Ad}$ & $\mathrm{O}$ & Kamrul $180(\mathrm{JUH})$ \\
\hline Dendrobium lindleyi Steud. & Linrium & Herb, ep & wd & All beats & - & Kamrul $1190(\mathrm{JUH})$ \\
\hline Peristylus sp. & - & Herb, e & wd & $\mathrm{Ka}$ & - & Kamrul 1585 (JUH) \\
\hline $\begin{array}{l}\text { Vanda tessellata (Roxb.) Hook. ex } \\
\text { G.Don. }\end{array}$ & Rasna & Herb, ep & wd & Ad & M & Kamrul 584 (JUH) \\
\hline
\end{tabular}

LEGEND: Habit. cl = climbing, $\mathrm{cr}=$ creeping, de = decumbent, ep = epiphytic, e = erect, er = erect reed, $1=$ large, $\mathrm{m}=$ medium, $\mathrm{pr}=$ prostrate, $\mathrm{ps}=$ parasitic, $\mathrm{aq}=$ aquatic, $\mathrm{s}=$ small, $\mathrm{sc}=$ scandent, $\mathrm{vi}=$ vine. HABITAT . cu $=$ cultivated, $\mathrm{fe}=$ forest margin, $\mathrm{fv}=$ forest valley, $\mathrm{gl}=$ grassland, $\mathrm{ht}=$ hill top, $\mathrm{hs}=$ hill slope, $\mathrm{ml}=$ marginal land, $\mathrm{pl}$ $=$ planted, $\mathrm{rb}=$ river bank, $\mathrm{rs}=$ roadsides, $\mathrm{sj}=$ scrub jungle, $\mathrm{wd}=$ in forest. Distrib. $=$ Distribution. Ad $=$ Adampur beat, $\mathrm{Ka}=$ Kamarchara beat, $\mathrm{Ku}=$ Kurma beat. Use $\mathrm{Co}=$ cosmetics, $\mathrm{Dy}=$ dye, $\mathrm{Du}=$ domestic uses, $\mathrm{Fb}=\mathrm{Fibre}, \mathrm{Fd}$ $=$ fodder, $\mathrm{Fr}=$ Fruit, $\mathrm{Fw}=$ fuel wood, $\mathrm{Gm}=$ green manure, $\mathrm{I}=$ insecticide, $\mathrm{Ju}=$ juice, $\mathrm{M}=$ medicine, $\mathrm{O}=$ ornamental, $\mathrm{Ol}=$ oil, $\mathrm{Pe}=$ perfume, $\mathrm{Pu}=$ pulse, $\mathrm{St}=$ shade tree, $\mathrm{Sb}=$ soil binder, $\mathrm{Sp}=$ spice, $\mathrm{Sw}=$ soft wood, $\mathrm{T}=$ timber, and V = vegetable. RSE = Representative Specimens Examined. * = Species listed in Red Data Book of Vascular Plants of Bangladesh.

In respect to land area, the number of angiosperm species found in RRF during this study seems higher than that reported by few studies on the protected areas of Bangladesh, e.g., Teknaf Game Reserve (Khan et al., 1994), and Chunuti Wildlife Sanctuary (Khan and Huq, 2001). In contrast, the number of angiosperm species found in RRF is lower than that of some other protected areas of the country, e.g., Rema-Kalenga Wildlife Sanctuary (Uddin and Hassan, 2004), and Lawachara National Park (Uddin and Hassan, 2010). These data indicate that this reserve forest houses an important portion of the flora of Bangladesh. 
The study recognizes a total of 387 angiosperm species of RRF as economically useful and among these species 82 are useful in two and 17 in three categories. The major categories of these economically useful species are medicinal (231 species), timber (50 species), fruit (49 species), ornamental (47 species), vegetable (34 species), fodder (23 species), domestic uses (19 species), fuel wood (11 species), and soil binder ( 9 species).

The RRF area houses 25 species included as threatened in the Red Data Book of Vascular Plants of Bangladesh (Table 1; Khan et al., 2001; Ara et al., 2013). This emumeration of threatened species is higher in respect to that reported for few forest areas, e.g., Lawachara National Park (Uddin and Hassan, 2010) and Sundarban Mangrove Forest (Rahman et al., 2016), and on the other hand, lower in respect to that recorded from other forest areas of Bangladesh (e.g., Rema-Kalenga forest; Uddin and Hassan, 2004). All of these 25 species were found in many localities of RRF with normal natural regeneration and any threat or stress exclusive for these species could not be recognized there, and therefore, they were categorized under the Lower Risk (LR) category for Rajkandi Reserve Forest.

This checklist provides basic information on all angiosperm species currently occurring in the Rajkandi Reserve Forest, which can be considered as an important database as well as baseline to track the trend of changes in the floristic composition of this reserve forest in course of time and different biogeographical processes. This study also informs the current status of 25 threatened species in RRF. These data might be useful in planning, management, conservation and sustainable development of this valuable forest resource of Bangladesh.

\section{Acknowledgements}

The authors are grateful to the authorities of the Bangladesh Forest Department, Bangladesh National Herbarium (DACB) and Dhaka University Salar Khan Herbarium (DUSH) for their cooperation during conducting this study. The authors are thankful to the Chief Editor and the Reviewers of the Journal for their critical review of the manuscript.

\section{References}

Ahmed, Z.U., Begum, Z.N.T., Hassan, M.A., Khondker, M., Kabir, S.M.H., Ahmed, M., Ahmed, A.T.A., Rahman, A.K.T. and Haque, E.U. (Eds) 2008-2009. Encyclopedia of Flora and Fauna of Bangladesh, Vols. 6-10, 11, 12. Asiatic Society of Bangladesh, Dhaka.

Alam, M.K. 1988. Annotated checklist of woody flora of Sylhet forests. Bulletinn 5, Plant Taxonomy Series. Bangladesh Forest Research Institute, Chittagong, pp. 1-153.

Ara, H., Khan, B. and Uddin, S.N. (Eds) 2013. Red Data Book of Vascular Plants of Bangladesh. Bangladesh National Herbarium, Dhaka, pp. 1-280.

Arefin, M.K., Rahman, M.M., Uddin, M.Z. and Hassan, M.A. 2011. Angiosperm flora of Satchari National Park, Habiganj, Bangladesh. Bangladesh J. Plant Taxon. 18(2): 117-140.

Bridson, D.M. and Forman, F. 1989. In: Bridson, D.M. and Forman, F. (Eds), The Herbarium Handbook. Royal Botanic Gardens, Kew, 214 pp.

Cronquist, A. 1981. An integrated system of classification of flowering plants. Columbia University Press, New York, pp. 1-1262.

Das, D.K. 1968. The vegetation of Sylhet forests. Pak. J. Forest. 18(3): 307-316.

Ghani, A. 1998. Medicinal Plants of Bangladesh with Chemical Constituents and Uses. Asiatic Society of Bangladesh, pp. 1-467.

Huq, A.M. 1986. Plant Names of Bangladesh. Bangladesh National Herbarium, BARC, Dhaka, Bangladesh, pp. 1-289. 
Haque, A.K.M.K., Khan, S.A., Uddin, S.N. and Rahim, M.A. 2016. Taxonomic checklist of the pteridophytes of Rajkandi Reserve Forest, Moulvibazar, Bangladesh. Jahangirnagar University J. Biol. Sci. 5(2): 27-40.

Hooker, J.D. 1872-1897. The Flora of British India. Vols. 1-7. L. Reeve \& Co., Ashford, Kent, UK.

IUCN. 2001. IUCN Red List Categories and Criteria. Version 3.1. IUCN, Gland, Switzerland and Cambridge, U.K.

Kanjilal, U.N., Kanjilal, P.C. and Das, A. 1934. Flora of Assam. Vol. 1. (Reprint 1982). A Von Book Company, Delhi.

Kanjilal, U.N., Kanjilal, P.C. and Das, A. 1938-1940. Flora of Assam. Vol. 2-4. (Reprint 1982). A Von Book Company, Delhi.

Khan, M.S., Rahman, M.M. and Ali, M.A. (Eds) 2001. Red Data Book of Vascular Plants of Bangladesh, Vol. 1. Bangladesh National Herbarium, Dhaka, Bangladesh, pp. 1-179.

Khan, M.S. and Huq, A.M. 2001. The vascular flora of Chunati wildlife sanctuary in south Chittagong. Bangladesh J. Plant Taxon. 8(1): 47-64.

Khan, M.S., Rahman, M.M., Huq. A.M., Mia, M.M.K. and Hassan, M.A. 1994. Assessment of Biodiversity of Teknaf Game Reserve in Bangladesh focussing on economically and ecologically important plant species. Bangladesh J. Plant Taxon. 1(1): 21-33.

Myers, N., Mittermeier, R.A., Mittermeier, C.G., da Fonseca, G.A.B. and Kent, J. 2000. Biodiversity hotspots for conservation priorities. Nature 403: 853-858.

Pasha, M.K and Uddin, S.B. 2013. Dictionary of Plant Names of Bangladesh (Vascular Plants). Janokalyan Prokashani, Chittagong, pp. 1-320.

Prain, D. 1903. Bengal Plants. Vols. 1 \& 2. (Reprint 1963). Botanical Survey of India, Calcutta.

Rahman, M.S., Hossain, M.G., Khan, S.A. and Uddin, S.N. 2016. An annotated checklist of the vascular plants of Sundarban mangrove forest of Bangladesh. Bangladesh J. Plant Taxon. 22(1): 17-41.

Singh, H.B. and Subramaniam, B. 2008. Field Manual on Herbarium Techniques. National Institute of Science Communication and Information Resources, pp. 1-297.

Sobuj, N.A, and Rahman, M. 2011. Assessment of plant diversity in Khadimnagar National Park of Bangladesh. International J. Environ. Sci. 2(1): 79-91.

The Plant List, 2013. The Plant List, a working list of all plant species. Version $1.1<\mathrm{http} / / / \mathrm{www}$.theplantlist.org/>. Accessed on 29 October 2017.

TROPICOS, 2017. Tropicos.org. <www.tropicos.org>. Missouri Botanical Garden, Saint Louis, Missouri, USA. Accessed on 20 October 2017.

Uddin, M.Z. and Hassan, M.A. 2004. Flora of Rema-Kalenga Wildlife Sanctuary. IUCN Bangladesh Country Office, Dhaka, Bangladesh, pp. 1-120.

Uddin, M.Z. and Hassan, M.A. 2010. Angiosperm diversity of Lawachara National Park (Bangladesh): A preliminary assessment. Bangladesh J. Plant Taxon. 17(1): 9-22.

van Valkenburg, J.L.C.H. and Bunyapraphatsara. N. (Eds) 2002. Plant Resources of South-East Asia, No. 12(2). Medicinal and Poisonous Plants 2. Prosea Foundation, Bogor, Indonesia, 782 pp.

Wu, Z.Y. and Raven, P.H. (Eds) 1994-2001. Flora of China, Vols. 8, 15-18, 24. Missouri Botanical Garden Press, St. Louis, USA.

Wu, Z.Y., Raven, P.H. and Hong, D.Y. (Eds) 1999-2013. Flora of China, Vols. 2-7, 9-14, 19-23, 25. Missouri Botanical Garden Press, St. Louis. 